The Annals of Applied Probability

2001, Vol. 11, No. 4, 1263-1291

\title{
ASYMPTOTIC ANALYSIS AND EXTINCTION IN A STOCHASTIC LOTKA-VOLTERRA MODEL
}

\author{
By F. C. KLEBANER ${ }^{1}$ AND R. LIPTSER ${ }^{1}$ \\ University of Melbourne and Tel Aviv University
}

\begin{abstract}
A stochastic Lotka-Volterra model is formulated by using the semimartingale approach. The large deviation principle is established, and is used to obtain a bound for the asymptotics of the time to extinction of prey population. The bound is given in terms of past-dependent ODEs closely related to the dynamics of the deterministic Lotka-Volterra model.
\end{abstract}

\section{Introduction. Main result.}

1.1. Deterministic Lotka-Volterra system. The Lotka-Volterra system of ordinary differential equations [Lotka (1925) and Volterra (1926)],

$$
\begin{aligned}
& \dot{x}_{t}=\mathfrak{a} x_{t}-\mathfrak{b} x_{t} y_{t}, \\
& \dot{y}_{t}=\mathfrak{c} x_{t} y_{t}-\mathfrak{b} y_{t},
\end{aligned}
$$

with positive $x_{0}, y_{0}$ and positive parameters $\mathfrak{a}, \mathfrak{b}, \mathfrak{c}, \mathfrak{D}$ describes a behavior of a predator-prey system in terms of the prey and predator "intensities" $x_{t}$ and $y_{t}$. Here, $\mathfrak{a}$ is the rate of increase of prey in the absence of predator, $\mathbb{D}$ is a rate of decrease of predator in the absence of prey while the rate of decrease in prey is proportional to the number of predators $\mathfrak{b} y_{t}$, and similarly the rate of increase in predator is proportional to the number of prey $c x_{t}$ [see, e.g., May (1976)]. The system (1.1) is one of the simplest nonlinear systems.

Since the population numbers are discrete, a description of the predatorprey model in terms of continuous intensities $x_{t}, y_{t}$ is based implicitly on a natural assumption that the numbers of both populations are large, and the intensities are obtained by a normalization of population numbers by a large parameter $K$. Thus (1.1) is an approximation, an asymptotic description of the interaction between the predator and prey. Although this model may capture some essential elements in that interaction, it is not suitable to answer questions of extinction of populations, as the extinction never occurs in the deterministic model; see Figure 1 for the pair $x_{t}, y_{t}$ in the phase plane.

We introduce here a probabilistic model which has as its limit the deterministic Lotka-Volterra model, evolves in continuous time according to the same local interactions and allows evaluating asymptotically the time for extinction of prey species.

There is a vast amount of literature on the Lotka-Volterra model, and a history of research on stochastic perturbations of this system both exact, approx-

Received June 2000; revised January 2001.

${ }^{1}$ Supported in part by Australian Research Council.

AMS 2000 subject classifications. Primary 60I27, 60F10.

Key words and phrases. Predator-prey models, large deviations, semimartingales, extinction 


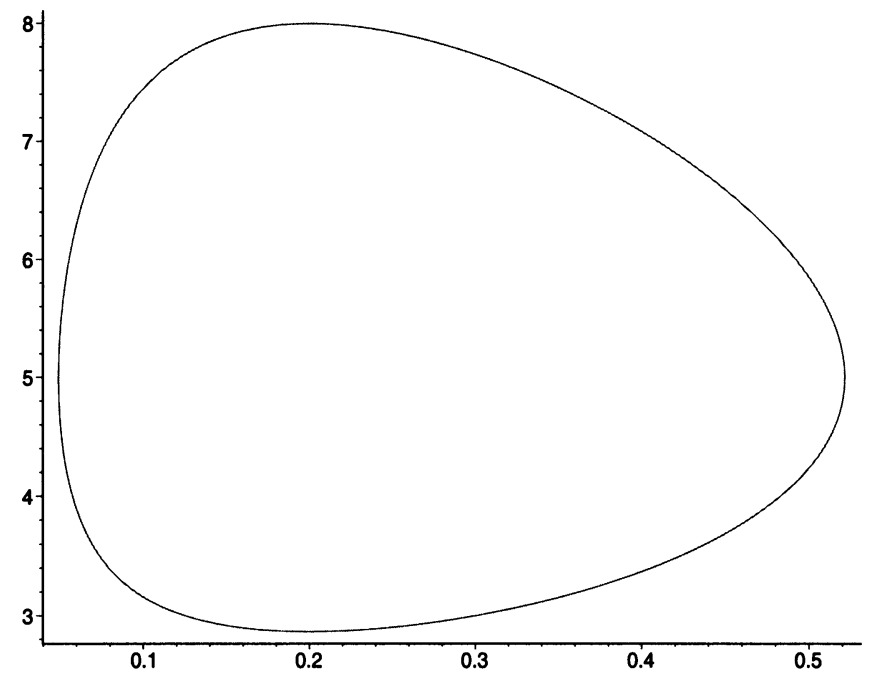

FIG. 1. $\quad r(x, y)$ when $\mathfrak{a}=5, \mathfrak{b}=1, \mathfrak{c}=5, \mathfrak{d}=1$.

imate and numerical; see, for example, Goel, Maitra and Montroll (1971), Turelli (1977), Kesten and Ogura (1981), Hitchcock (1986), Watson (1987), Roozen (1989) and references therein. We approach the problem of extinction via the theory of large deviations, thus obtaining new theoretical results, which previously were studied numerically.

The system (1.1) possesses the first integral which is a closed orbit in the first quadrant of phase plane $x, y$. It is given by

$$
r(x, y)=c x-\mathfrak{b} \log x+\mathfrak{b} y-\mathfrak{a} \log y+r_{0},
$$

where $r_{0}$ is an arbitrary constant. It depends only on the initial points $\left(x_{0}, y_{0}\right)$ (see Figure 1).

1.2. Stochastic Lotka-Volterra system. In this paper, we introduce and analyze a probabilistic model of prey-predator population related to the classical Lotka-Volterra equations.

Let $X_{t}$, and $Y_{t}$ be numbers of prey and predators at time $t$. We start with simple balance equations for prey-predator populations

$$
\begin{aligned}
& X_{t}=X_{0}+\pi_{t}^{\prime}-\pi_{t}^{\prime \prime}, \\
& Y_{t}=Y_{0}+\hat{\pi}_{t}^{\prime}-\hat{\pi}_{t}^{\prime \prime}
\end{aligned}
$$

where:

$\pi_{t}^{\prime}$ is a number of prey born up to time $t$.

$\pi_{t}^{\prime \prime}$ is a number of prey killed up to time $t$.

$\hat{\pi}_{t}^{\prime}$ is a number of predators born up to time $t$.

$\hat{\pi}_{t}^{\prime}$ is a number of predators died up to time $t$. 
We assume that $\pi_{t}^{\prime}, \pi_{t}^{\prime \prime}, \hat{\pi}_{t}^{\prime}, \hat{\pi}_{t}^{\prime \prime}$, are double Poisson processes with the following random rates: $\mathfrak{a} X_{t}, \frac{\mathfrak{b}}{K} X_{t} Y_{t}, \frac{\mathfrak{c}}{K} X_{t} Y_{t}, \triangleright Y_{t}$, respectively and disjoint jumps [the latter assumption reflects the fact that in a short time interval $(t, t+\delta t)$ only one prey might be born and only one might be killed, only one predator might be born and only one might die, with the above-mentioned intensities; moreover all these events are disjoint in time].

The existence of such a model is not obvious; therefore in Section 2 we give its detailed probabilistic derivation.

Assume $X_{0}=K x_{0}$ and $Y_{0}=K x_{0}$ for some fixed positive $x_{0}, y_{0}$ and a large integer parameter $K$. Introduce the normalized by $K$ prey and predator populations

$$
x_{t}^{K}=\frac{X_{t}}{K} \quad \text { and } \quad y_{t}^{K}=\frac{Y_{t}}{K} .
$$

In terms of $x_{t}^{K}$ and $y_{t}^{K}$ the introduced intensities for double Poisson processes can be written as

$$
K\left(\mathfrak{a} x_{t}^{K}, \mathfrak{b} x_{t}^{K} y_{t}^{K}, \mathfrak{c} x_{t}^{K} y_{t}^{K}, \mathfrak{b} y_{t}^{K}\right) .
$$

We justify the choice of the probabilistic model given in (1.3) by Theorem 2 which states that the solution of the Lotka-Volterra equations is the limit (in probability) for $\left(x_{t}^{K}, y_{t}^{K}\right)$,

$$
\left(x_{t}^{K}, y_{t}^{K}\right) \rightarrow\left(x_{t}, y_{t}\right), \quad K \rightarrow \infty .
$$

Such an approximation is known as the fluid approximation. Results on the fluid approximation for Markov discontinuous processes can be found in Kurtz (1981) and are adapted to the case considered here, despite that in our case the two intensities do not satisfy the linear growth condition in $x_{t}^{K}, y_{t}^{K}$.

1.3. Fomulation of the problem. Here, we are interested in evaluation of the prey extinction, namely, the asymptotics in $K$ of

$$
P\left(T_{\mathrm{ext}}^{K} \leq T, \inf _{t \leq T} y_{t}^{K}>0\right)=0,
$$

where $T_{\text {ext }}^{K}=\inf \left\{t>0: x_{t}^{K}=0\right\}$ is the prey extinction time. Unfortunately, the fluid approximation does not provide much information on the extinction time $T_{\text {ext }}^{K}$. Since for $x_{0}>0, y_{0}>0$ the "fluid limit" $\left(x_{t}, y_{t}\right)$ remains positive for any $t>0$ (see Figure 1), we have

$$
\lim _{K \rightarrow \infty} P\left(T_{\text {ext }}^{K} \leq T, \inf _{t \leq T} y_{t}^{K}>0\right)=0 .
$$

A historical comment on $T_{\text {ext }}^{K}$ of evaluation is due. There is a large amount of literature on the subject of extinction mostly using some simplification of the original problem, such as linearization, and numerical studies. For a somewhat different model with a state-dependent noise, Hitchcock (1986) showed that ultimate extinction is certain, and derived exact probabilities for the predators to become eventually extinct when the prey birth rate is zero. A power series 
approximation for the extinction probabilities as well as the number of steps to extinction in special cases were given. Numerical studies of probabilities of extinction were also done in Smith and Mead (1979, 1980), and Watson (1987) where a rough approximation (based on the normal approximation) was also given.

Due to (1.4), the rate of convergence in (1.4) is of interest, and is the subject of this paper. Neither fluid nor even diffusion approximations for the stretched differences

$$
\sqrt{K}\left(x_{t}^{K}-x_{t}\right), \sqrt{K}\left(y_{t}^{K}-y_{t}\right)
$$

are effective for such analysis.

Freidlin (1998), Freidlin and Weber (1998) carried out an effective asymptotic analysis for randomly perturbed oscillators and other Hamiltonian systems. Their approach is based on the approximation of the first integral process [in our case $r\left(x_{t}^{K}, y_{t}^{K}\right)$ ] by a scalar diffusion. However, in our case this approach does not seem to be of use, since at the time of extinction the first integral process $r\left(x_{t}^{K}, y_{t}^{K}\right)$ explodes; see (1.2).

A large deviation (LD) type evaluation yields results in our case. The random process $\left(x_{t}^{K}, y_{t}^{K}\right)$ is a vector semimartingale, so that it appears that one can have the large deviation principle (LDP) by using a general results from Pukhaskii (1999). However, the method from Pukhaskii does not serve the model studied here, since the intensities of two double Poisson processes are of quadratic form in $x_{t}^{K}, y_{t}^{K}$. For the same reason we could not find adequate methods for proving the (LDP) in the literature, for example, Wentzell (1989), Dupuis and Ellis (1997), Freidlin and Wentzel (1984), Dembo and Zeitouni (1993).

It is well known that the main helpful tool in verification of the LDP with "unbounded intensities," satisfying a linear growth condition, is the exponential negligibility for sets $\left\{\sup _{t \leq T} x_{t}^{K} \geq C\right\},\left\{\sup _{t \leq T} y_{t}^{K} \geq C\right\}$, for large $K, C$ and every $T>0$. In our case two from four intensities do not satisfy the linear growth condition. Nevertheless, specifics of the model and the fact that $x_{t}^{K}, y_{t}^{K}$ are nonnegative processes with bounded jumps allow establishing the above-mentioned exponential negligibility (Lemma 2) and deriving the LDP similarly to Liptser and Pukhalskii (1992).

Although in principle the LDP allows finding the logarithmic rate with norming $\frac{1}{K}$ for

$$
P\left(T_{\mathrm{ext}}^{K} \leq T, \inf _{t \leq T} y_{t}^{K}>0\right)
$$

a realistic procedure for determining the required rate depends heavily on the structure of the LDP rate function. In our case we deal with purely discontinuous process and the rate function is extremely inconvenient for this purpose; therefore, we restrict ourselves to finding only a lower bound for the required rate. 


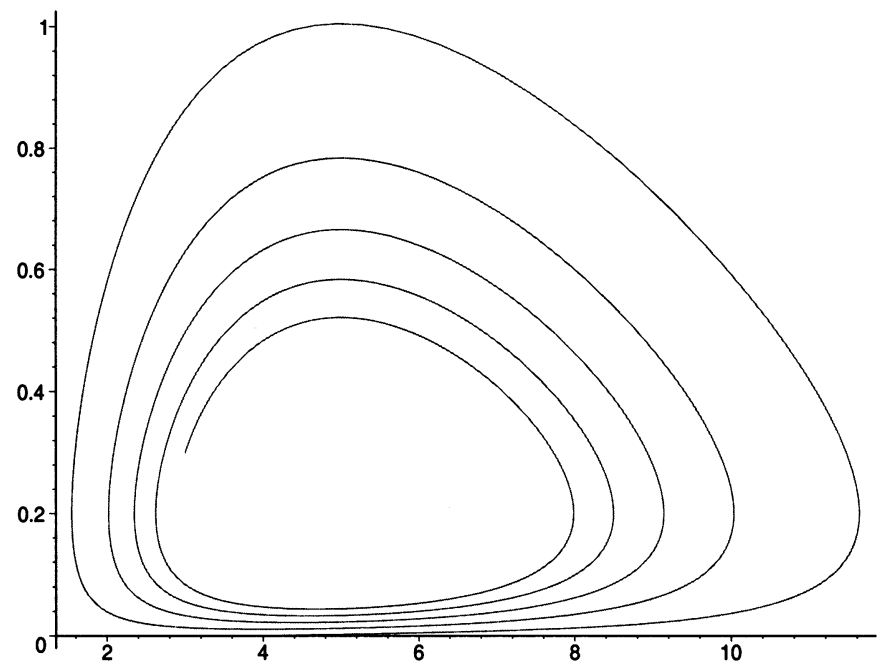

FIG. 2. Trajectory that attains the lower bound. Parameters are the same as for Figure 1.

1.4. Main result. To formulate the main result, we need to introduce a system of past-dependent differential equations parameterized by $q>0$,

$$
\begin{aligned}
& \dot{\phi}_{t}^{q}=\phi_{t}^{q}\left(\mathfrak{a}-\mathfrak{b} \psi_{t}^{q}\right)-q e^{-\int_{0}^{t}\left(\mathfrak{a}-\mathfrak{b} \psi_{t}^{q}\right) d s} \sqrt{\mathfrak{b} \phi_{t}^{q} \psi_{t}^{q}}, \\
& \dot{\psi}_{t}^{q}=\psi_{t}^{q}\left(\mathfrak{c} \phi_{t}^{q}-\mathfrak{b}\right),
\end{aligned}
$$

subject to the initial condition $\psi_{0}^{q}=y_{0}, \phi_{0}^{q}=x_{0}$, and denote

$$
T^{q}=\inf \left\{t>0: \phi_{t}^{q}=0\right\} .
$$

THEOREM 1. For every $T>0$,

$$
\begin{gathered}
\liminf _{K \rightarrow \infty} \frac{1}{K} \log P\left(T_{\mathrm{ext}}^{K} \leq T, \inf _{t \leq T} y_{t}^{K}>0\right) \\
\geq-\frac{x_{0}^{2}}{2 \int_{0}^{T^{q_{*}}} e^{-2 \int_{0}^{t}\left(\mathfrak{a}-\mathfrak{b} \psi_{t}^{q_{*}}\right) d s} \mathfrak{b} \phi_{t}^{q_{*}} \psi_{t}^{q_{*}} d t},
\end{gathered}
$$

where $T^{q_{*}}$ is associated with the smallest $q=q_{*}$ for which $T^{q_{*}} \leq T$.

1.5. Example. We give here an example with $\mathfrak{a}=5, \mathfrak{b}=1, \mathfrak{c}=5, \mathfrak{b}=1$ and $T=15$. The trajectories for $\phi_{t}^{q}, \psi_{t}^{q}$, on which the lower bound is attainable, are given in the phase plane (see Figure 2). Parameter $q_{*} \approx 0.0023$ and $T^{q_{*}} \approx 15$, while the value of the rate function defined later in (4.2) is $J_{*}\left(\phi^{q_{*}}, \psi^{q_{*}}\right) \approx$ 0.0018 .

1.6. Remark. We can show for a stochastic Lotka-Volterra model with a different noise structure (much simpler and somewhat artificial) the exact asymptotic relation in Theorem 1 (rather than a lower bound), as well as that the minimum of the rate function in the LDP occurs on the solution similar 
to (1.5). Therefore, loosely speaking, (1.5) (see Figure 2) gives a likely path to extinction in the stochastic Lotka-Volterra model.

The article is organized as follows. In Section 2 we give description of the stochastic model, in Section 3 we show the fluid approximation, in Section 4 we formulate the LDP and prove the main result. The verification of the LDP, which is quite technical, is done in the Appendix.

\section{The model: description of stochastic dynamics.}

2.1. Existence. In this section, we show that the random process $\left(X_{t}, Y_{t}\right)$ is well defined by (1.3). To this end, let us introduce four independent sequences of processes,

$$
\begin{aligned}
& \Pi_{t}^{\mathfrak{a}}=\left(\Pi_{t}^{\mathfrak{a}}(1), \Pi_{t}^{\mathfrak{a}}(2), \ldots\right), \\
& \Pi_{t}^{\mathfrak{b} / K}=\left(\Pi_{t}^{\mathfrak{b} / K}(1), \Pi_{t}^{\mathfrak{b} / K}(2), \ldots\right), \\
& \Pi_{t}^{\mathfrak{c} / K}=\left(\Pi_{t}^{\mathfrak{c} / K}(1), \Pi_{t}^{\mathfrak{c} / K}(2), \ldots\right), \\
& \Pi_{t}^{\mathfrak{d}}=\left(\Pi_{t}^{\mathfrak{d}}(1), \Pi_{t}^{\mathfrak{d}}(2), \ldots\right) .
\end{aligned}
$$

Each of them is a sequence of i.i.d. Poisson processes characterized by rates $\mathfrak{a}, \frac{\mathfrak{b}}{K}, \frac{\mathfrak{c}}{K}, \mathfrak{d}$, respectively. Define the processes $\left(X_{t}, Y_{t}\right)$ by the system of Itô equations

$$
\begin{aligned}
& X_{t}=X_{0}+\int_{0}^{t} \sum_{n \geq 1} I\left(X_{s-} \geq n\right) d \Pi_{s}^{\mathfrak{a}}(n)-\int_{0}^{t} \sum_{n \geq 1} I\left(X_{s-} Y_{s-} \geq n\right) d \Pi_{s}^{\mathfrak{b} / K}(n), \\
& Y_{t}=Y_{0}+\int_{0}^{t} \sum_{n \geq 1} I\left(X_{s-} Y_{s-} \geq n\right) d \Pi_{s}^{\mathrm{c} / K}(n)-\int_{0}^{t} \sum_{n \geq 1} I\left(Y_{s-} \geq n\right) d \Pi_{s}^{\mathfrak{d}}(n),
\end{aligned}
$$

governed by these Poisson processes, which obviously has a unique solution on the time interval $\left[0 ; T_{\infty}\right)$, where

$$
T_{\infty}=\inf \left\{t>0: X_{t} \vee Y_{t}=\infty\right\} .
$$

The double Poisson processes involved in (1.3) are obtained then in the following way:

$$
\begin{aligned}
& \pi_{t}^{\prime}=\int_{0}^{t} \sum_{n \geq 1} I\left(X_{s-} \geq n\right) d \Pi_{s}^{\mathfrak{a}}(n), \\
& \pi_{t}^{\prime \prime}=\int_{0}^{t} \sum_{n \geq 1} I\left(X_{s-} Y_{s-} \geq n\right) d \Pi_{s}^{\mathfrak{b} / K}(n), \\
& \hat{\pi}_{t}^{\prime}=\int_{0}^{t} \sum_{n \geq 1} I\left(X_{s-} Y_{s-} \geq n\right) d \Pi_{s}^{\mathrm{c} / K}(n), \\
& \hat{\pi}_{t}^{\prime \prime}=\int_{0}^{t} \sum_{n \geq 1} I\left(Y_{s-} \geq n\right) d \Pi_{s}^{\delta}(n) .
\end{aligned}
$$


Let us show that $\pi_{t}^{\prime}, \pi_{t}^{\prime \prime}, \hat{\pi}_{t}^{\prime}, \hat{\pi}_{t}^{\prime \prime}$ defined in (2.2) satisfy the required properties. Since all Poisson processes are independent, their jumps are disjoint, so that the jumps of $\pi_{t}^{\prime}, \pi_{t}^{\prime \prime}, \hat{\pi}_{t}^{\prime}, \hat{\pi}_{t}^{\prime \prime}$ are disjoint as well. To describe structure of intensities for $\pi_{t}^{\prime}, \pi_{t}^{\prime \prime}, \hat{\pi}_{t}^{\prime}, \hat{\pi}_{t}^{\prime \prime}$, let us introduce a stochastic basis $(\Omega, \mathscr{T}, \mathbf{F}=$ $\left.\left(\mathscr{F}_{t}\right)_{t>0}, P\right)$ supplied by the filtration $\mathbf{F}$ generated by all Poisson processes and satisfying the general conditions. Then, obviously, the random process

$$
A_{t}^{\prime}=\int_{0}^{t} \sum_{n \geq 1} I\left(X_{s} \geq n\right) \mathfrak{a} d s
$$

is the compensator of $\pi_{t}^{\prime}$. On the other hand, since $X_{s}$ is an integer-valued random variable, we have $\sum_{n \geq 1} I\left(X_{s} \geq n\right)=X_{s}$; that is, $A_{t}^{\prime}=\int_{0}^{t} a X_{s} d s$ and the intensity of $\pi_{t}^{\prime}$ is $a X_{t}$. Analogously, others compensators are seen to be

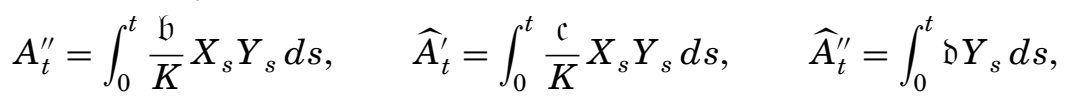

and thus all other intensities have the required form.

We now show that the process $\left(X_{t}, Y_{t}\right)_{t \geq 0}$ does not explode.

LEMMA 1.

$$
P\left(T_{\infty}=\infty\right)=1
$$

Proof. Set $T_{n}^{X}=\inf \left\{t>0: X_{t} \geq n\right\}, n \geq 1$ and denote by $T_{\infty}^{X}=$ $\lim _{n \rightarrow \infty} T_{n}^{X}$. Due to (2.1) it holds that

$$
E X_{t \wedge T_{n}^{X}} \leq X_{0}+\int_{0}^{t} a E X_{s \wedge T_{n}^{X}} d s
$$

and so, by the Gronwall-Bellman inequality $E X_{T_{n}^{X \wedge T}} \leq X_{0} e^{a T}$ for every $T>0$. Hence by the Fatou lemma $E X_{T_{\infty}^{X} \wedge T} \leq X_{0} e^{\mathfrak{a} T}$. Consequently,

$$
P\left(T_{\infty}^{X} \leq T\right)=0 \quad \forall T>0 .
$$

Set $T_{\ell}^{Y}=\inf \left\{\mathrm{t}: Y_{t} \geq \ell\right\}, \ell \geq 1$ and denote by $T_{\infty}^{Y}=\lim _{\ell \rightarrow \infty} T_{\ell}^{Y}$. Due to (2.1) it holds that

$$
\begin{aligned}
E Y_{t \wedge T_{n}^{X} \wedge T_{t}^{Y}} & \leq Y_{0}+\int_{0}^{t} \frac{\mathfrak{c}}{K} E\left(X_{s \wedge T_{n}^{X} \wedge T_{\ell}^{Y}} Y_{s \wedge T_{n}^{X} \wedge T_{\ell}^{Y}}\right) d s \\
& \leq Y_{0}+\int_{0}^{t} \frac{\mathfrak{c}}{K} n E Y_{s \wedge T_{n}^{X} \wedge T_{\ell}^{Y}} d s .
\end{aligned}
$$

Hence, by the Gronwall-Bellman inequality for every $T>0$ we have $E Y_{T \wedge T_{n}^{x} \wedge T_{\ell}^{y}} \leq Y_{0} e^{c n T}$ and by the Fatou lemma,

$$
E Y_{T \wedge T_{n}^{X} \wedge T_{\infty}^{Y}} \leq Y_{0} e^{(\mathrm{c} / K) n T}, \quad n \geq 1 .
$$

Consequently, $P\left(T_{\infty}^{Y} \leq T_{n}^{X} \wedge T\right)=0, \forall T>0, n \geq 1$ and, since $T_{n}^{X} \nearrow \infty$, $n \rightarrow \infty$, we obtain

$$
P\left(T_{\infty}^{Y} \leq T\right)=0 \quad \forall T>0 .
$$

Since $T_{\infty}=T_{\infty}^{X} \wedge T_{\infty}^{Y}, P\left(T_{\infty} \leq T\right)=P\left(T_{\infty}^{X} \leq T\right)+P\left(T_{\infty}^{Y} \leq T\right)$, and we have $P\left(T_{\infty} \leq T\right)=0$ for any $T>0$. 
Corollary 1. For $T_{n}=\inf \left\{t: X_{t} \vee Y_{t} \geq n\right\}$,

$$
\lim _{n \rightarrow \infty} P\left(T_{n} \leq T\right)=0 \quad \forall T>0 .
$$

The above description of the model allows claiming that $\left(X_{t}, Y_{t}\right)$ is a continuous-time pure jump Markov process with jumps of two possible sizes in both coordinates: " 1 " and " -1 " and infinitesimal transition probabilities (as $\delta t \rightarrow 0)$,

$$
\begin{aligned}
P\left(X_{t+\delta t}=X_{t}+1 \mid X_{t}, Y_{t}\right) & =\mathfrak{a} X_{t} \delta t+o(\delta t), \\
P\left(X_{t+\delta t}=X_{t}-1 \mid X_{t}, Y_{t}\right) & =\frac{\mathfrak{b}}{K} X_{t} Y_{t} \delta t+o(\delta t), \\
P\left(Y_{t+\delta t}=Y_{t}+1 \mid X_{t}, Y_{t}\right) & =\frac{\mathfrak{c}}{K} X_{t} Y_{t} \delta t+o(\delta t), \\
P\left(Y_{t+\delta t}=Y_{t}-1 \mid X_{t}, Y_{t}\right) & =\grave{b} Y_{t} \delta t+o(\delta t) .
\end{aligned}
$$

2.2. Semimartingale description for $\left(x_{t}^{K}, y_{t}^{K}\right)$. Let $A_{t}^{\prime}, A_{t}^{\prime \prime}, \widehat{A}_{t}^{\prime}, \widehat{A}_{t}^{\prime \prime}$ be the compensators of $\pi_{t}^{\prime}, \pi_{t}^{\prime \prime}, \hat{\pi}_{t}^{\prime}, \hat{\pi}_{t}^{\prime \prime}$ defined above. Introduce martingales

$$
M_{t}^{\prime}=\pi_{t}^{\prime}-A_{t}^{\prime}, \quad M_{t}^{\prime \prime}=\pi_{t}^{\prime \prime}-A_{t}^{\prime \prime}, \quad \widehat{M}_{t}^{\prime}=\hat{\pi}_{t}^{\prime}-\widehat{A}_{t}^{\prime}, \quad \widehat{M}_{t}^{\prime \prime}=\hat{\pi}_{t}^{\prime \prime}-\widehat{A}_{t}^{\prime \prime},
$$

and also normalized martingales

$$
m_{t}^{K}=\frac{M_{t}^{\prime}-M_{t}^{\prime \prime}}{K} \quad \text { and } \quad \hat{m}_{t}^{K}=\frac{\widehat{M}_{t}^{\prime}-\widehat{M}_{t}^{\prime \prime}}{K} .
$$

Then, from (1.3) it follows that the process $\left(x_{t}^{K}, y_{t}^{K}\right)$ admits the semimartingale decomposition

$$
\begin{aligned}
& x_{t}^{K}=x_{0}+\int_{0}^{t}\left[\mathfrak{a} x_{s}^{K}-\mathfrak{b}_{s}^{K} x_{s}^{K} y_{s}^{K}\right] d s+m_{t}^{K}, \\
& y_{t}^{K}=y_{0}+\int_{0}^{t}\left[\mathfrak{c} x_{s}^{K} y_{s}^{K}-\mathfrak{b} y_{s}^{K}\right] d s+\hat{m}_{t}^{K},
\end{aligned}
$$

which is a stochastic analogue (in integral form) of (1.1)

In the sequel we need quadratic variations of the martingales in (2.4) and (2.5). It is well known [see, e.g., Liptser and Shiryaev (1998), Chapter 18 or Klebaner (1998), Theorem 9.3] that all martingales are locally square integrable and possess the predictable quadratic variations

$$
\left\langle M^{\prime}\right\rangle_{t}=A_{t}^{\prime}, \quad\left\langle M^{\prime \prime}\right\rangle_{t}=A_{t}^{\prime \prime} \quad \text { and } \quad\left\langle\widehat{M}^{\prime}\right\rangle_{t}=\widehat{A}_{t}^{\prime}, \quad\left\langle\widehat{M}^{\prime \prime}\right\rangle_{t}=\widehat{A}_{t}^{\prime \prime},
$$

and zero mutual predictable quadratic variations $\left\langle M^{\prime}, M^{\prime \prime}\right\rangle_{t} \equiv 0, \ldots$ $\left\langle\widehat{M}^{\prime}, \widehat{M}^{\prime \prime}\right\rangle_{t} \equiv 0$, implied by the disjointness of jumps for $\pi_{t}^{\prime}, \pi_{t}^{\prime \prime}, \hat{\pi}_{t}^{\prime}, \hat{\pi}_{t}^{\prime \prime}$. 
Hence

$$
\begin{aligned}
\left\langle m^{K}, \hat{m}^{K}\right\rangle_{t} & \equiv 0, \\
\left\langle m^{K}\right\rangle_{t} & =\frac{1}{K} \int_{0}^{t}\left(\mathfrak{a} x_{s}^{K}+\mathfrak{b} x_{s}^{K} y_{s}^{K}\right) d s, \\
\left\langle\hat{m}^{K}\right\rangle_{t} & =\frac{1}{K} \int_{0}^{t}\left(\mathfrak{c} x_{s}^{K} y_{s}^{K}+\mathfrak{b} y_{s}^{K}\right) d s .
\end{aligned}
$$

2.3. Stochastic exponential and cumulant function. In the large deviation theory we use stochastic exponential, and to this end the following representation is more convenient:

$$
\begin{aligned}
& x_{t}^{K}=x_{0}=\frac{\pi_{t}^{\prime}}{K}-\frac{\pi_{t}^{\prime \prime}}{K}, \\
& y_{t}^{K}=y_{0}=\frac{\hat{\pi}_{t}^{\prime}}{K}-\frac{\hat{\pi}_{t}^{\prime \prime}}{K} .
\end{aligned}
$$

$\frac{\pi_{t}^{\prime}}{K}, \frac{\pi_{t}^{\prime \prime}}{K}, \frac{\hat{\pi}_{t}^{\prime}}{K}, \frac{\hat{\pi}_{t}^{\prime \prime}}{K}$ are counting process with jumps of the unit size $K^{-1}$ and compensators $\frac{A_{t}^{\prime}}{K}, \frac{A_{t}^{\prime \prime}}{K}, \frac{\widehat{A}_{t}^{\prime}}{K}, \frac{\widehat{A}_{t}^{\prime \prime}}{K}$, respectively. With every pair $\left(\frac{\pi_{t}^{\prime}}{K}, \frac{A_{t}^{\prime}}{K}\right), \ldots,\left(\frac{\hat{\pi}_{t}^{\prime \prime}}{K}, \frac{\widehat{A}_{t}^{\prime \prime}}{K}\right)$ and a predictable process $\nu(t)$ such that for any $T>0$ and $K$ large enough,

$$
\int_{0}^{T} e^{|\nu(t)| / K}\left(x_{t}^{K}+y_{t}^{K}+x_{t}^{K} y_{t}^{K}\right) d t<\infty, \quad P \text {-a.s. }
$$

we associate nonnegative processes

$$
\begin{aligned}
& z_{t}^{\prime}\left(\frac{\nu}{K}\right)=\exp \left(\int_{0}^{t} \frac{\nu(s)}{K} d \pi_{s}^{\prime}-\left(e^{\nu(s) / K}-1\right) d A_{s}^{\prime}\right), \\
& \ldots \ldots \ldots \ldots \ldots \ldots \ldots \ldots \ldots \ldots \ldots \ldots \ldots \ldots \ldots \ldots \ldots \ldots \ldots \ldots \ldots \ldots \ldots \ldots \ldots \ldots \ldots \ldots \ldots \ldots \ldots \ldots \ldots \ldots \ldots \ldots \ldots \ldots \ldots \\
& \hat{z}_{t}^{\prime \prime}\left(\frac{\nu}{K}\right)=\exp \left(\int_{0}^{t} \frac{\nu(s)}{K} d \hat{\pi}_{s}^{\prime \prime}-\left(e^{\nu(s) / K}-1\right) d \widehat{A}_{s}^{\prime}\right) .
\end{aligned}
$$

Applying the Itô formula to $z_{t}^{\prime}\left(\frac{\nu}{K}\right)$, we find

$$
d z_{t}^{\prime}\left(\frac{\nu}{K}\right)=z_{t-}^{\prime}\left(\frac{\nu}{K}\right)\left(e^{\nu(t) / K}-1\right) d\left(\pi_{t}^{\prime}-A_{t}^{\prime}\right),
$$

that is, $z_{t}^{\prime}\left(\frac{\nu}{K}\right)$ is a local martingale [analogously $z_{t}^{\prime \prime}\left(\frac{\nu}{K}\right), \hat{z}_{t}^{\prime}\left(\frac{\nu}{K}\right), \hat{z}_{t}^{\prime \prime}\left(\frac{\nu}{K}\right)$ are local martingales as well]. All these martingales are nonnegatives, so that they are supermartingales [see Problem 1.4.4 in Liptser and Shiryayev (1989) or Theorem 7.20 in Klebaner (1998)]. Hence for any Markov time $\tau$ we have $E z_{\tau}^{\prime}\left(\frac{\nu}{K}\right) \leq 1, \ldots, E \hat{z}_{t}^{\prime \prime}\left(\frac{\nu}{K}\right) \leq 1$.

Introduce also (for predictable processes $\lambda$ and $\mu$ )

$$
z_{t}^{K}(\lambda, \mu)=z_{t}^{\prime}\left(\frac{\lambda}{K}\right) z_{t}^{\prime \prime}\left(-\frac{\lambda}{K}\right) \hat{z}_{t}^{\prime}\left(\frac{\mu}{K}\right) \hat{z}_{t}^{\prime \prime}\left(-\frac{\mu}{K}\right) .
$$


Applying the Itô formula to $z_{t}^{K}(\lambda, \mu)$ and taking into account that jumps of $\pi_{t}^{\prime}, \pi_{t}^{\prime \prime}, \hat{\pi}_{t}^{\prime}, \hat{\pi}_{t}^{\prime \prime}$ are disjoint we find

$$
\begin{aligned}
d Z_{t}^{K}(\lambda, \mu)=z_{t-}^{K}(\lambda, \mu)\left\{\left(e^{\lambda(t) / K}-1\right) d\left(\pi_{t}^{\prime}-A_{t}^{\prime}\right)+\left(e^{(\lambda(t) / K)}-1\right) d\left(\pi_{t}^{\prime \prime}-A_{t}^{\prime \prime}\right)\right. \\
\left.+\left(e^{\mu(t) / K}-1\right) d\left(\hat{\pi}_{t}^{\prime}-\widehat{A}_{t}^{\prime}\right)+\left(e^{(\mu(t) / K)}-1\right) d\left(\hat{\pi}_{t}^{\prime \prime}-\widehat{A}_{t}^{\prime \prime}\right)\right\},
\end{aligned}
$$

so that $Z_{t}^{K}(\lambda, \mu)$ is a positive local martingale as well as a supermartingale with

$$
E Z_{\tau}^{K}(\lambda, \mu) \leq 1
$$

for any Markov time $\tau$.

Set

$$
\begin{aligned}
& G_{\phi}(\lambda ; u, v)=\lambda(\mathfrak{a}-\mathfrak{b} v) u+\left(e^{\lambda}-1-\lambda\right) \mathfrak{a} u+\left(e^{-\lambda}-1+\lambda\right) \mathfrak{b} u v, \\
& G_{\psi}(\mu ; u, v)=\mu(\mathfrak{c} u-\mathfrak{b}) v+\left(e^{\mu}-1-\mu\right) \mathfrak{c} u v+\left(e^{-\mu}-1+\mu\right) \mathfrak{d} v .
\end{aligned}
$$

and define the so-called cumulant function,

$$
G(\lambda, \mu ; u, v)=G_{\phi}(\lambda ; u, v)+G_{\psi}(\mu ; u, v) .
$$

Using the cumulant function, (2.10) can be written as

$$
\begin{aligned}
Z_{t}^{K}(\lambda, \mu)= & \exp \left\{\int_{0}^{t}\left(\lambda(s) d x_{s}^{K}+\int_{0}^{t} \mu(s) d y_{s}^{K}\right)\right\} \\
& \times \exp \left\{-\int_{0}^{t} K G\left(\frac{\lambda(s)}{K}, \frac{\mu(s)}{K}, x_{s}^{K}, y_{s}^{K}\right) d s\right\} .
\end{aligned}
$$

Hence an equivalent multiplicative decomposition holds for the exponential semimartingale

$$
\exp \left(\int_{0}^{t}\left(\lambda(s) d x_{s}^{K}+\mu(s) d y_{s}^{K}\right)\right)=Z_{t}^{K}(\lambda, \mu) V_{t}^{K}(\lambda, \mu)
$$

with the positive local martingale $Z_{t}^{K}(\lambda, \mu)$ and a positive predictable process

$$
V_{t}^{K}(\lambda, \mu)=\exp \left\{\int_{0}^{t} K G\left(\frac{\lambda(s)}{K}, \frac{\mu(s)}{K}, x_{s}^{K}, y_{s}^{K}\right) d s\right\} .
$$

3. Fluid approximation. In this section we justify the choice of the stochastic dynamics by showing that the Lotka-Volterra equations describe a limit (fluid approximation) for the family

$$
\left(x_{t}^{K}, y_{t}^{K}\right), K \nearrow \infty .
$$

In what follows $\left(x_{t}, y_{t}\right)$ is a solution of the Lotka-Volterra equation (1.1).

THEOREM 2. For any $T>0$ and $\eta>0$,

$$
\lim _{K \rightarrow \infty} P\left(\sup _{t \leq T}\left(\left|x_{t}^{K}-x_{t}\right|+\left|y_{t}^{K}-y_{t}\right|\right)>\eta\right)=0 .
$$


Proof. Set

$$
T_{n}^{K}=\inf \left\{t: x_{t}^{K} \vee y_{t}^{K} \geq n\right\} .
$$

Since $T_{n}^{K}=T_{n K}$ (see Corollary 1 to Lemma 1$)$,

$$
\lim _{n \rightarrow \infty} \limsup _{K \rightarrow \infty} P\left(T_{n}^{K} \leq T\right)=0 .
$$

Hence, it suffices to show that for every $n \geq 1$,

$$
\lim _{K \rightarrow \infty} P\left(\sup _{t \leq T_{n}^{K} \wedge T}\left(\left|x_{t}^{K}-x_{t}\right|+\left|y_{t}^{K}-y_{t}\right|\right)>\eta\right)=0 .
$$

Since $\sup _{t \leq T_{n}^{K} \wedge T}\left(x_{t}^{K} \vee y_{t}^{K}\right) \leq n+1$, there is a constant $L_{n}$, depending on $n$ and $T$, such that for $t \leq T_{n}^{K} \wedge T$,

$$
\begin{gathered}
\left|\left(\mathfrak{a} x_{t}^{K}-\mathfrak{b} x_{t}^{K} y_{t}^{K}\right)-\left(\mathfrak{a} x_{t}-\mathfrak{b} x_{t} y_{t}\right)\right| \leq L_{n}\left(\left|x_{t}^{K}-x_{t}\right|+\left|y_{t}^{K}-y_{t}\right|\right), \\
\left|\left(\mathfrak{c} x_{t}^{K} y_{t}^{K}-\mathfrak{b} y_{t}^{K}\right)-\left(\mathfrak{c} x_{t} y_{t}-\mathfrak{b} y_{t}\right)\right| \leq L_{n}\left(\left|x_{t}^{K}-x_{t}\right|+\left|y_{t}^{K}-y_{t}\right|\right) .
\end{gathered}
$$

These inequalities and (1.1), (2.4) imply

$$
\begin{aligned}
& \left|x_{T_{n}^{K} \wedge T}^{K}-x_{T_{n}^{K} \wedge T}\right|+\left|y_{T_{n}^{K} \wedge T}^{K}-y_{T_{n}^{K} \wedge T}\right| \\
& \leq 2 L_{n} \int_{0}^{t}\left(\left|x_{s \wedge T_{n}^{K}}^{K}-x_{s \wedge T_{n}^{K}}\right|+\left|y_{s \wedge T_{n}^{K}}^{K}-y_{s \wedge T_{n}^{K}}\right|\right) d s \\
& \quad+\sup _{t \leq T_{n}^{K} \wedge T}\left|m_{t}^{K}\right|+\sup _{t \leq T_{n}^{K} \wedge T}\left|\hat{m}_{t}^{K}\right| .
\end{aligned}
$$

Now, by the Gronwall-Bellman inequality we find

$$
\sup _{t \leq T_{n}^{K} \wedge T}\left(\left|x_{t}^{K}-x_{t}\right|+\left|y_{t}^{K}-y_{t}\right|\right) \leq e^{2 L_{n} T}\left(\sup _{t \leq T_{n}^{K} \wedge T}\left|m_{t}^{K}\right|+\sup _{t \leq T_{n}^{K} \wedge T}\left|\hat{m}_{t}^{K}\right|\right) .
$$

Therefore (3.3) holds, if both $\sup _{t \leq T_{n}^{K} \wedge T}\left|m_{t}^{K}\right|$ and $\sup _{t \leq T_{n}^{K} \wedge T}\left|\hat{m}_{t}^{K}\right|$ converge in probability to zero as $K \rightarrow \infty$. By the Doob inequality for martingales (see, e.g., Theorem 1.9.1(3) and Problem 1.9.2 in Liptser and Shiryayev (1989) the required convergence takes place provided that both $\left\langle m^{K}\right\rangle_{T_{n}^{K} \wedge T}$ and $\left\langle\hat{m}^{K}\right\rangle_{T_{n}^{K} \wedge T}$ converge to zero in probability as $K \rightarrow \infty$. But by (2.7) $\left\langle m^{K}\right\rangle_{T_{n}^{K} \wedge T} \leq \frac{\text { const. }}{K}$, $\left\langle\hat{m}^{K}\right\rangle_{T_{n}^{K} \wedge T} \leq \frac{\text { const. }}{K}$, and the proof is complete.

\section{Formulation of the LDP and the proof of Theorem 1.}

4.1. $L D P$. The random process $\left(x_{t}^{K}, y_{t}^{K}\right)_{t \geq 0}$ has nonnegative paths from the Skorokhod space $\mathbb{D}^{2}=\mathbb{D}_{[0, \infty)}^{2}$. Since we are going to apply the LDP for asymptotic analysis of the extinction time on a finite interval $[0, T]$, we examine the LDP in $\mathbb{D}_{[0, T]}^{2}$. Because of the fluid approximation, the limit for $\left(x_{t}^{K}, y_{t}^{K}\right)_{t \leq T}$ is $\left(x_{t}, y_{t}\right)_{t \leq T}$ with continuous differentiable functions $x_{t}$ and $y_{t}$. 
This fact allows examining the LDP in the metric space $\left(\mathbb{D}_{[0, T]}^{2}, \varrho\right)$ supplied by the uniform metric $\varrho:\left(x^{\prime}, x^{\prime \prime} \in \mathbb{D}_{[0, T]}^{2}\right)$ and

$$
\varrho\left(x^{\prime}, x^{\prime \prime}\right)=\sum_{j=1}^{2} \sup _{t \leq T}\left|x_{t}^{\prime}(j)-x_{t}^{\prime \prime}(j)\right| .
$$

More exactly, since $x_{t}^{K} \geq 0, y_{t}^{K} \geq 0$ and

$$
\tau_{x}^{K}=\inf \left\{t>0: x_{t}^{K}=0\right\}, \quad \tau_{y}^{K}=\inf \left\{t>0: y_{t}^{K}=0\right\}
$$

are also absorption points for processes $x_{t}^{K}$ and $y_{t}^{K}$, respectively, let us introduce a subspace $\mathbb{D}_{[0, T]}^{2,+}$ of nonnegative function from $\mathbb{D}_{[0, T]}^{2}$. It is obvious that $\mathbb{D}_{[0, T]}^{2,+}$ is a closed subset of $\mathbb{D}_{[0, T]}^{2}$ in the metric $\varrho$.

Therefore we formulate the LDP in metric space $\left(\mathbb{D}_{[0, T]}^{2,+}, \varrho\right)$.

THEOREM 3. For every $T>0$, the family $\left(x_{t}^{K}, y_{t}^{K}\right)_{t \leq T}, K \nearrow \infty$ obeys the LDP in the metric space $\left(\mathbb{D}_{[0, T]}^{2,+}, \varrho\right)$ with the rate of speed $\frac{1}{K}$ and the (good) rate function

$$
J_{T}(\phi, \psi)=\left\{\begin{array}{lr}
\int_{0}^{T} \sup \left(\lambda \dot{\phi}_{t}+\mu \dot{\psi}_{t}-G\left(\lambda, \mu ; \phi_{t}, \psi_{t}\right)\right) d t, & \left.\right|_{d \phi_{t} \ll d t, d \psi_{t} \ll d t} \\
\phi_{0}=x_{0}, \psi_{0}=y_{0} & \\
\infty, & \text { otherwise } .
\end{array}\right.
$$

The proof of the LDP is given in the Appendix.

4.2. Proof of Theorem 1. Denote by $\Gamma^{o}$ the interior of the set

$$
\left\{(\phi, \psi) \in \mathbb{D}_{[0, T]}^{2,+}: \inf _{t \leq T} \phi_{t}=0, \inf _{t \leq T} \psi_{t}>0\right\} .
$$

Due to the LDP,

$$
\liminf _{K \rightarrow \infty} \frac{1}{K} \log P\left(\inf _{t \leq T} x_{t}^{K}=0, \inf _{t \leq T} y_{t}^{K}>0\right) \geq-\inf _{(\phi, \psi) \in \Gamma^{\phi}} J_{T}(\phi, \psi) .
$$

It is clear that

$$
\left.\inf _{(\phi, \psi) \in \Gamma^{o}} J_{T}(\phi, \psi) \leq \inf _{(\phi, \psi) \in \Gamma^{o}} J_{T}(\phi, \psi)\right),
$$

where $\Gamma^{\phi}$ is a subset of $\Gamma^{o}$ of absolutely continuous functions $\phi_{t}, \psi_{t}$ with $\phi_{0}=x_{0}, \psi_{0}=y_{0}$ and for any $\phi=\left(\phi_{t}\right)_{t \leq T}$,

$$
\dot{\psi}_{t}=\psi_{t}\left(\mathfrak{c} \phi_{t}-\mathfrak{D}\right) \text {. }
$$

To emphasize the fact that $\psi_{t}$ is a solution of (4.4) we use the notation $\psi_{t}^{\phi}$. The function $\psi_{t}^{\phi}$ remains positive for any finite time interval and moreover,

$$
\begin{aligned}
\sup _{\lambda, \mu} & \left\{\lambda \dot{\phi}_{t}+\mu \dot{\psi}_{t}^{\phi}-G\left(\lambda, \mu ; \phi_{t}, \psi_{t}^{\phi}\right)\right\}=\sup _{\lambda}\left\{\lambda \dot{\phi}_{t}-G_{\phi}\left(\lambda ; \phi_{t}, \psi_{t}^{\phi}\right)\right\} \\
= & \sup _{\lambda}\left\{\lambda\left(\dot{\phi}_{t}-\phi_{t}\left(\mathfrak{a}-\mathfrak{b} \psi_{t}^{\phi}\right)\right)-\left(e^{\lambda}-1-\lambda\right) \mathfrak{a} \phi_{t}-\left(e^{-\lambda}-1+\lambda\right) \mathfrak{b} \phi_{t} \psi_{t}^{\phi}\right\} .
\end{aligned}
$$


Taking into account that functions $\left(\phi_{t}\right)$ 's from $\Gamma^{\phi}$ have to be absorbed on $[0, T]$, we choose a subset $\Gamma_{<0}^{\phi}$ of $\Gamma^{\phi}$ with functions $\phi_{t}$ 's satisfying the property

$$
\dot{\phi}_{t}-\phi_{t}\left(\mathfrak{a}-\mathfrak{b} \psi_{t}^{\phi}\right)<0 \text {. }
$$

In this case, we claim

$$
\begin{aligned}
& \sup _{\lambda \in \mathbb{R}}\left\{\lambda\left(\dot{\phi}_{t}-\phi_{t}\left(\mathfrak{a}-\mathfrak{b} \psi_{t}^{\phi}\right)\right)-\left(e^{\lambda}-1-\lambda\right) \mathfrak{a} \phi_{t}-\left(e^{-\lambda}-1+\lambda\right) \mathfrak{b} \phi_{t} \psi_{t}^{\phi}\right\} \\
& =\sup _{\lambda<0}\left\{\lambda\left(\dot{\phi}_{t}-\phi_{t}\left(\mathfrak{a}-\mathfrak{b} \psi_{t}^{\phi}\right)\right)-\left(e^{\lambda}-1-\lambda\right) \mathfrak{a} \phi_{t}-\left(e^{-\lambda}-1+\lambda\right) \mathfrak{b} \phi_{t} \psi_{t}^{\phi}\right\} \\
& \leq \sup _{\lambda<0}\left\{\lambda\left(\dot{\phi}_{t}-\phi_{t}\left(\mathfrak{a}-\mathfrak{b} \psi_{t}^{\phi}\right)\right)-\left(e^{-\lambda}-1+\lambda\right) \mathfrak{b} \phi_{t} \psi_{t}^{\phi}\right\} \\
& \leq \sup _{\lambda<0}\left\{\lambda\left(\dot{\phi}_{t}-\phi_{t}\left(\mathfrak{a}-\mathfrak{b} \psi_{t}^{\phi}\right)\right)-\frac{\lambda^{2}}{2} \mathfrak{b} \phi_{t} \psi_{t}^{\phi}\right\} \\
& \quad= \begin{cases}\frac{\left(\dot{\phi}_{t}-\phi_{t}\left(\mathfrak{a}-\mathfrak{b} \psi_{t}^{\phi}\right)\right)^{2}}{2 \mathfrak{b} \phi_{t} \psi_{t}^{\phi}}, & \phi_{t}>0\left(\text { excluding absorption point of } \phi_{t}\right), \\
0, & \phi_{t}=0 .\end{cases}
\end{aligned}
$$

For a square integrable on $[0, T]$ and nonnegative function $u(t)$ introduce a system of differential euations

$$
\begin{aligned}
\dot{\phi}_{t} & =\phi_{t}\left(\mathfrak{a}-\mathfrak{b} \psi_{t}\right)-u(t) \sqrt{\mathfrak{b} \phi_{t} \psi_{t}^{\phi}}, \\
\dot{\psi}_{t}^{\phi} & =\psi_{t}^{\phi}\left(\mathfrak{c} \phi_{t}-\mathfrak{b}\right),
\end{aligned}
$$

subject to $\phi_{0}=x_{0}, \psi_{0}^{\phi}=y_{0}$. We use now an obvious inequality

$$
\inf _{(\phi, \psi) \in \Gamma^{o}} J_{T}(\phi, \psi) \leq \inf _{u(t): \inf _{t \leq T} \phi_{t}=0} \frac{1}{2} \int_{0}^{T} u^{2}(t) d t .
$$

Thus we get

$$
\begin{gathered}
\liminf _{K \rightarrow \infty} \frac{1}{K} \log P\left(\inf _{t \leq T} x_{t}^{K}=0, \inf _{t \leq T} y_{t}^{K}>0\right) \\
\geq-\inf _{u(t): \inf _{t \leq T} \phi_{t}=0} \frac{1}{2} \int_{0}^{T} u^{2}(t) d t .
\end{gathered}
$$

We now specify the $\inf _{u(t): \inf _{t \leq T} \phi_{t}=0} \frac{1}{2} \int_{0}^{T} u^{2}(t) d t$.

Set $T^{\prime}=\inf \left\{t: \phi_{t}=0\right\}$ and show that $u(t)$ exists so that $T^{\prime} \leq T$. Since $\phi_{t} \geq 0$, it holds $\psi_{t}^{\phi} \geq y_{0} e^{-\mathfrak{D} T}$ and thereby

$$
\dot{\phi}_{t} \leq \mathfrak{a} \sqrt{\phi_{t}}\left(\sqrt{\phi_{t}}-u(t) \frac{\sqrt{\mathfrak{b} e^{-\mathfrak{\delta} T}}}{\mathfrak{a}}\right) .
$$

With $r=\frac{\sqrt{\mathfrak{b e}^{-\triangleright T} T}}{\mathfrak{a}} u(t) \equiv \ell>\frac{\sqrt{x_{0}}}{r}$ and $p(\ell)=-\mathfrak{a}\left(\sqrt{x_{0}}-\ell r\right)$ we have

$$
\dot{\phi}_{t} \leq-p(\ell) \sqrt{\phi_{t}} \text {, }
$$


that is, $\sqrt{\phi_{t}} \leq \sqrt{x_{0}}-p(\ell) t$. Now, for any $T^{\prime}>0$, the choice $p(\ell)=\frac{\sqrt{x_{0}}}{T^{\prime}}$ guarantees $T^{\prime} \leq T$.

If some $u(t)$ is chosen and associated with $T^{\prime}<T$, it is obvious that $u(t) \equiv 0$ for $t \in\left[T^{\prime}, T\right]$. Then, particularly, in (4.6) the integral $\int_{0}^{T} u^{2}(t) d t$ is replaced by $\int_{0}^{T^{\prime}} u^{2}(t) d t$. On the other hand, due to $\phi_{T^{\prime}}=0$ we have

$$
0=e^{\int_{0}^{T^{\prime}}\left(\mathfrak{a}-\mathfrak{b} \psi_{s}^{\phi}\right) d s}\left(x_{0}-\int_{0}^{T^{\prime}} e^{-\int_{0}^{t}\left(\mathfrak{a}-\mathfrak{b} \psi_{s}^{\phi}\right) d s} u(t) \sqrt{\mathfrak{b} \phi_{t} \psi_{t}^{\phi}} d t\right)
$$

that is, $x_{0}=\int_{0}^{T^{\prime}} e^{-\int_{0}^{t}\left(\mathfrak{a}-\mathfrak{b} \psi_{s}^{\phi}\right)} d s u(t) \sqrt{\mathfrak{b} \phi_{t} \psi_{t}^{\phi}} d t$. Further, the Cauchy-Schwarz inequality implies

$$
\int_{0}^{T^{\prime}} u^{2}(t) d t \geq \frac{x_{0}^{2}}{\int_{0}^{T^{\prime}} e^{-2 \int_{0}^{t}\left(\mathfrak{a}-\mathfrak{b} \psi_{s}^{\phi}\right) d s} \mathfrak{b} \phi_{t} \psi_{t}^{\phi} d t}
$$

Take any positive $q$ and $u(t)=q e^{-\int_{0}^{t}\left(\mathfrak{a}-\mathfrak{b} \psi_{s}^{\phi}\right)} d s \sqrt{\mathfrak{b} \phi_{t} \psi_{t}^{\phi}}$ then the inequality given in (4.7) becomes an equality, and only for

$$
q^{\prime}=\frac{x_{0}}{\int_{0}^{T^{\prime}} e^{-2 \int_{0}^{t}\left(\mathfrak{a}-\mathfrak{b} \psi_{s}^{\phi}\right) d s} \mathfrak{b} \phi_{t} \psi_{t}^{\phi} d t} .
$$

we have $\phi_{T^{\prime}}=0$.

Let $\left(q, T^{q}\right)$ be a pair such that (1.6) holds with $q^{\prime}$ and $T^{\prime}$ replaced by $q$ and $T^{q}$ respectively and $T^{q} \leq T$. Denote by $\phi_{t}^{q}$ and $\psi_{t}^{q}$ the solution of (1.5) on $\left[0, T_{q}\right]$ corresponding to the pair $\left(q, T^{q}\right)$. Then we get the lower bound

$$
\begin{aligned}
& \liminf _{K \rightarrow \infty} \frac{1}{K} \log P\left(T_{\mathrm{ext}}^{K} \leq T, \inf _{t \leq T} y_{t}^{K}>0\right) \\
& \geq-\inf \frac{x_{0}^{2}}{2 \int_{0}^{T_{q}} e^{-2 \int_{0}^{t}\left(\mathfrak{a}-\mathfrak{b} \psi_{s}^{\phi}\right) d s} u(t) \mathfrak{b} \phi_{t}^{q} \psi_{t}^{q} d t},
\end{aligned}
$$

where "inf" is taken over all $T_{q} \leq T$.

The final step of the proof uses the property

$$
q^{\prime}<q^{\prime \prime} \Longrightarrow \frac{x_{0}^{2}}{\int_{0}^{T^{q^{\prime}}} e^{-2 \int_{0}^{t}\left(\mathfrak{a}-\mathfrak{b} \psi_{s}^{q^{\prime}}\right) d s} \mathfrak{b} \phi_{t}^{q^{\prime}} \psi^{q^{\prime}} d t}<\frac{x_{0}^{2}}{\int_{0}^{T_{q^{\prime \prime}}} e^{-2 \int_{0}^{t}\left(\mathfrak{a}-\mathfrak{b} \psi_{s}^{q^{\prime \prime}}\right) d s} \mathfrak{b} \phi_{t}^{q^{\prime \prime}} \psi_{t}^{q^{\prime \prime}} d t},
$$

implied by (4.8). Consequently $q$ has to be chosen as small as possible with $T_{q} \leq T$. 


\section{APPENDIX}

\section{Verification of LDP.}

A.1. Preliminaries. We will follow here Liptser and Pukhalskii (1992). By their Theorem 1.3, the family

$$
\left(x_{t}^{K}, y_{t}^{K}\right)_{t \leq K}, K \nearrow \infty
$$

obeys the LDP in the metric space $\left(\mathbb{D}_{[0, T]}^{2}, \varrho\right)$ with the rate of speed $\frac{1}{K}$ and the rate function $J_{T}$ given in (4.2) provided that this family is $C$-exponentially tight and obeys $C$-local LDP. Due to Pukhalskii (1991), adapted to the case considered, $C$-exponential tightness holds, if

$$
\begin{aligned}
& \lim _{C \rightarrow \infty} \limsup _{K \rightarrow \infty} \frac{1}{K} \log P\left(\sup _{t \leq T} x_{t}^{K}+\sup _{t \leq T} y_{t}^{K}>C\right)=-\infty, \\
& \lim _{\delta \rightarrow 0} \limsup _{K \rightarrow \infty} \frac{1}{K} \log \sup P\left(\sup _{t \leq \delta}\left(\left|x_{t+\tau}^{K}-x_{\tau}^{K}\right|+\sup _{t \leq \delta}\left|y_{t+\tau}^{K}-y_{\tau}^{K}\right|\right)>\eta\right) \\
& \quad=-\infty
\end{aligned}
$$

for any $\eta>0$, where "sup" is taken over all stopping times $\tau \leq T$. $C$ local LDP is valid, if for any $(\phi, \psi) \in \mathbb{D}_{[0, T]}^{2}$

$$
\limsup _{\delta \rightarrow 0} \limsup _{K \rightarrow \infty} \frac{1}{K} \log P\left(\varrho\left(\left(x^{K}, y^{K}\right),(\phi, \psi)\right) \leq \delta\right) \leq-J_{T}(\phi, \psi),
$$

$$
\liminf _{\delta \rightarrow 0} \liminf _{K \rightarrow \infty} \frac{1}{K} \log P\left(\varrho\left(\left(x^{K}, y^{K}\right),(\phi, \psi)\right) \leq \delta\right) \geq-J_{T}(\phi, \psi) .
$$

Since the linear growth condition for intensities of counting processes is lost, a verification of (A.1) requires a different technique from the one used in Liptser and Pukhalskii (1992). We verify (A.1) in the next subsection.

A.2. Exponential negligibility of $\sup _{t \leq T} x_{t}^{K}, \sup _{t \leq T} y_{t}^{K}$.

LEMMA 2. For every $T>0$,

$$
\begin{aligned}
& \lim _{L \rightarrow \infty} \limsup _{K \rightarrow \infty} \frac{1}{K} \log P\left(\sup _{t \leq T} x_{t}^{K} \geq L\right)=-\infty, \\
& \lim _{C \rightarrow \infty} \limsup _{K \rightarrow \infty} \frac{1}{K} \log P\left(\sup _{t \leq T} y_{t}^{K} \geq C\right)=-\infty .
\end{aligned}
$$


PROOF. Recall that $x_{t}^{K}=x_{0}+\frac{\pi_{t}^{\prime}-\pi_{t}^{\prime \prime}}{K}$ and $M_{t}^{\prime}=\pi_{t}^{\prime}-A_{t}^{\prime}$; that is, $d x_{t}^{K} \leq$ $\frac{d \pi_{t}^{\prime}}{K}=a x_{t}^{K} d t+\frac{d M_{t}^{\prime}}{K}$ and so $x_{t}^{K} \leq e^{\mathfrak{a} t}\left(x_{0}+\frac{1}{K} \int_{0}^{t} e^{-\mathfrak{a} s} d M_{s}^{\prime}\right)$. Consequently, the first statement of the lemma is valid provided that

$$
\lim _{L \rightarrow \infty} \limsup _{K \rightarrow \infty} \frac{1}{K} \log P\left(\sup _{t \leq T} \int_{0}^{t} e^{-\mathfrak{a} s} d M_{s}^{\prime} \geq K L\right)=-\infty .
$$

It is clear that with $\sigma_{L}^{K}=\inf \left\{t: \int_{0}^{t} e^{-a s} d M_{s}^{\prime} \geq K L\right\}$ it suffices to establish

$$
\lim _{L \rightarrow \infty} \limsup _{K \rightarrow \infty} \frac{1}{K} \log P\left(\sigma_{L}^{K} \leq T\right)=-\infty
$$

To this end, with $r>0$ and $\nu(s)=r e^{-a s}$ we define $z_{t}^{\prime}(\nu)$ by the first formula in (2.9) with $\frac{\nu(s)}{K}$ replaced by $\nu(s)$ and use an obvious modification of this formula,

$$
z_{t}^{\prime}(\nu)=\exp \left(\int_{0}^{t} \nu(s) d M_{s}^{\prime}-\int_{0}^{t}\left(e^{\nu(s)}-1-\nu(s)\right) d A_{s}^{\prime}\right) .
$$

Recall that $z_{t}^{\prime}(\nu)$ is a supermartingale with $E{z^{\prime}}_{\sigma_{L}^{K} \wedge T}^{\prime}(\nu) \leq 1$. We use this inequality for the next one,

$$
1 \geq E z_{\sigma_{L}^{K} \wedge T}^{\prime}(\nu) I\left(\sigma_{L}^{K} \geq T\right) .
$$

We sharpen that inequality, by evaluating below ${z_{\sigma_{L}^{K} \wedge T}^{\prime}}^{(\nu)}$ on the set $\left\{\sigma_{L}^{K} \leq T\right\}$,

$$
\begin{aligned}
\log {z^{\prime}}_{\sigma_{L}^{K} \wedge T}^{\prime}(\nu) & =r \int_{0}^{\sigma_{L}^{K} \wedge T} e^{-\mathfrak{a s}} d M_{s}^{\prime}-\int_{0}^{\sigma_{L}^{K} \wedge T}\left(e^{r e^{-a s}}-1-r e^{-\mathfrak{a} s}\right) K \mathfrak{a} x_{s}^{K} d s \\
& \geq r K L-\int_{0}^{\sigma_{L}^{K} \wedge T}\left(e^{r}-1-r\right) K \mathfrak{a} x_{s}^{K} d s \\
& \geq r K L-\int_{0}^{T}\left(e^{r}-1-r\right) K \mathfrak{a} x_{s \wedge \sigma_{L}^{K}}^{K} d s .
\end{aligned}
$$

To continue this evaluation we find an upper bound for $x_{s \wedge \sigma_{L}^{K}}^{K}$. Since

$$
\int_{0}^{\sigma_{L}^{K} \wedge T} e^{-\mathfrak{a} s} d M^{\prime} s \leq K L+1
$$

(recall that jumps of $\int_{0}^{t} e^{-a s} d M^{\prime} s$ is bounded by 1 ), we claim

$$
x_{\sigma_{L}^{K} \wedge t}^{K} \leq e^{\mathfrak{a} t}\left(x_{0}+1+L\right) .
$$

Hence, with $p=\frac{e^{\mathrm{a} T}\left(x_{0}+1+L\right)}{L}$, we arrive at the lower bound

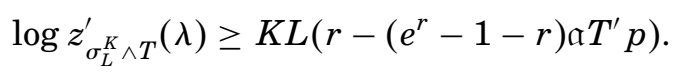

It is clear that $r^{\circ}>0$ can be chosen so that $r^{\circ}-\left(e^{r^{\circ}}-1-r^{\circ}\right) \mathfrak{a} T p:=\ell>0$ and therefore $\log z_{\sigma_{L}^{K} \wedge T}^{\prime}\left(\lambda^{\circ}\right) \geq K L \ell \quad(\ell>0)$. 
Thus, (A.7) with $\nu(s)=r e^{\mathfrak{a} s}$ replaced by $\nu^{\circ}(s) r^{\circ} e^{\mathfrak{a} s}$, implies

$$
\frac{1}{K} \log P\left(\sigma_{L}^{K} \leq T\right) \leq-L \ell \rightarrow-\infty, \quad L \rightarrow \infty,
$$

that is, (A.5) holds.

The proof of the second statement of the lemma uses (A.5) heavily. Since

$$
\left\{\sup _{t \leq T} y_{t}^{K} \geq C\right\} \subseteq\left\{\sup _{t \leq \sigma_{L}^{K} \wedge T} y_{t}^{K} \geq C\right\} \cup\left\{\sigma_{L}^{K} \leq T\right\}
$$

and thereby

$$
P\left(\sup _{t \leq T} y_{t}^{K} \geq C\right) \leq 2\left(P\left(\sup _{t \leq \sigma_{L}^{K} \wedge T} y_{t}^{K} \geq C\right) \bigvee P\left(\sigma_{L}^{K} \leq T\right)\right),
$$

by virtue of (A.5) it suffices to check that for every fixed $L$,

$$
\lim _{C \rightarrow \infty} \limsup _{K \rightarrow \infty} \frac{1}{K} \log P\left(\sup _{t \leq \sigma_{L}^{K} \wedge T} y_{t}^{K}+C\right)=-\infty .
$$

The verification of (A.8) is similar to the proof of the first statement of the lemma. It is clear that there is a positive constant $R_{L}$ so that $I\left(\sigma_{L}^{K} \geq t\right) x_{t}^{K} \leq$ $R_{L}, t \geq 0$. Further, due to $d y_{t}^{K} \leq \mathrm{c} x_{t}^{K} y_{t}^{K} d t+\frac{1}{K} d \widehat{M}_{t}^{\prime}$ we have

$$
\begin{aligned}
d y_{t \wedge \sigma_{L}^{K}}^{K} & \leq c I\left(\sigma_{L}^{K} \geq t\right) x_{t}^{K} y_{t}^{K} d t+\frac{1}{K} d \widehat{M}_{t \wedge \sigma_{L}^{K}}^{\prime} \\
& \leq c R_{L} y_{t}^{K} d t+\frac{1}{K} d \widehat{M}_{t \wedge \sigma_{L}^{K}}^{\prime}
\end{aligned}
$$

and hence $y_{t \wedge \sigma_{L}^{K}}^{K} \leq e^{c R_{L} t}\left(y_{0}+\int_{0}^{t \wedge \sigma_{L}^{K}} e^{-c R_{L} d \widehat{M}_{s}^{\prime}}\right)$. Now, introduce

$$
\tau_{L, C}^{K}=\inf \left\{t: \int_{0}^{t \wedge \sigma_{L}^{K}} e^{-c R_{L}} d \widehat{M}_{s}^{\prime} \geq C\right\}
$$

and note that (A.8) holds provided that

$$
\lim _{C \rightarrow \infty} \limsup _{K \rightarrow \infty} \frac{1}{K} \log P\left(\tau_{L, C}^{K} \leq T\right)=-\infty \quad \forall L>0 .
$$

The proof of (A.9) is similar to the proof of (A.5), so here we give only a sketch of it. Set $c^{\prime}=c R_{L}$ and for positive $r$ take $\nu(s)=r e^{-c^{\prime} s}$. Introduce a supermartingale

$$
\hat{z}_{t}^{\prime}(\nu)=\exp \left(\int_{0}^{t} \nu(s) d \widehat{M}_{s}^{\prime}-\int_{0}^{t}\left(e^{\nu(s)}-1-\nu(s)\right) d \widehat{A}_{s}^{\prime}\right) .
$$

Due to $E \hat{z}_{\sigma_{L}^{K} \wedge T_{L, C}^{K} \wedge T}^{\prime}(\nu) \leq 1$ write $1 \geq E \hat{z}_{\sigma_{L}^{K} \wedge T_{L, C}^{K} \wedge T}^{\prime}(\nu) I\left(\tau_{L, C}^{K} \leq T\right)$ and evaluate from below $\hat{z}_{\sigma_{L}^{K} \wedge T_{L, C}^{K} \wedge T}^{\prime}(\nu)$ on the set $\left\{\tau_{L, C}^{K} \leq T\right\}$ as

$$
\log \hat{z}_{\sigma_{L}^{K} \wedge T_{L, C}^{K} \wedge T}^{\prime K}(\nu) \geq K C\left(r-\left(e^{r}-1-r\right) \mathfrak{c}^{\prime} T p\right) .
$$


With $r^{\circ}$ such that $r^{\circ}-\left(e^{r^{\circ}}-1 r^{\circ}\right) c^{\prime} T p=: \ell>0$ similarly to the proof of the first statement of the lemma we obtain $\frac{1}{K} \log P\left(\tau_{L, C}^{K} \leq T\right) \leq-C \ell$ and (A.9) holds.

A.3. C-Exponential tightness. (A.1) is implied by Lemma 2.

To verify (A.2), let us note that

$$
\begin{aligned}
x_{t+\tau}^{K}-x_{\tau}^{K} & =\frac{1}{K}\left[\pi_{t+\tau}^{\prime}-\pi_{\tau}^{\prime}\right]+\frac{1}{K}\left[\pi_{t+\tau}^{\prime \prime}-\pi_{\tau}^{\prime \prime}\right], \\
y_{t+\tau}^{K}-y_{\tau}^{K} & =\frac{1}{K}\left[\hat{\pi}_{t+\tau}^{\prime}-\hat{\pi}_{\tau}^{\prime}\right]+\frac{1}{K}\left[\hat{\pi}_{t+\tau}^{\prime \prime}-\hat{\pi}_{\tau}^{\prime \prime}\right] .
\end{aligned}
$$

Evidently, it suffices to establish the validity of (A.2) for every

$$
\frac{1}{K}\left[\pi_{t+\tau}^{\prime}-\pi_{\tau}^{\prime}\right], \ldots, \frac{1}{K}\left[\hat{\pi}_{t+\tau}^{\prime \prime}-\hat{\pi}_{\tau}^{\prime \prime}\right]
$$

separately.

Recall that the Markov time $T_{n}^{K}$ is defined in (3.1). By virtue of Lemma 2 we claim that $\lim _{n \rightarrow \infty} \lim \sup _{K \rightarrow \infty} \frac{1}{K} \log P\left(T_{n}^{K} \leq T\right)=-\infty$. Hence, with any $n \geq 1$ we have to verify (A.2) only for

$$
\begin{aligned}
& \lim _{K \rightarrow \infty} \frac{1}{K} \log \sup _{\tau \leq T} P\left(\pi_{(\tau+\delta) \wedge T_{n}^{K}}^{\prime}-\pi_{\tau \wedge T_{n}^{K}}^{\prime}>K \eta\right)=0, \\
& \lim _{K \rightarrow \infty} \frac{1}{K} \log \sup _{\tau \leq T} P\left(\hat{\pi}_{(\tau+\delta) \wedge T_{n}^{K}}^{\prime \prime}-\hat{\pi}_{\tau \wedge T_{n}^{K}}^{\prime \prime}>K \eta\right)=0 .
\end{aligned}
$$

The proofs for the above relations are similar. So, we give below only one of them for $\pi_{t}^{\prime \prime}$. With $r>0$ set $\dot{\nu}(s)=r I_{\left(\tau \wedge T_{n}^{K},(\tau+\delta) \wedge T_{n}^{K}\right]}(s)$. The random process $\nu(s)$ is bounded and left continuous (and thereby predictable). Consequently [compare (2.9)], the random process

$$
z_{t}^{\prime \prime}(\nu)=\exp \left(\int_{0}^{t} \nu(s) d \hat{\pi}_{s}^{\prime \prime}-\int_{0}^{t}\left(e^{\nu(s)}-1\right) d A_{s}^{\prime \prime}\right)
$$

is a supermartingale with $E z_{T+\delta}^{\prime \prime}(\lambda) \leq 1$. Therefore,

$$
1 \geq E z_{T+\delta}^{\prime \prime}(\nu) I\left(\pi_{(\tau+\delta) \wedge T_{n}^{K}}^{\prime \prime}-\pi_{\tau \wedge T_{n}^{K}}^{\prime \prime}>K \eta\right) .
$$

Since $\left[A_{(\tau+\delta) \wedge T_{n}^{K}}^{\prime \prime}-A_{\tau \wedge T_{n}^{K}}^{\prime \prime}\right] \leq \delta \frac{\mathfrak{h}}{K}(n+1)^{2}$, on the set $\left\{\pi_{(\tau+\delta) \wedge T_{n}^{K}}^{\prime \prime}-\pi_{\tau \wedge T_{n}^{K}}^{\prime \prime}>K \eta\right\}$,

$$
\begin{aligned}
\log z_{T+\delta}^{\prime \prime}(\nu) & =r\left[\pi_{(\tau+\delta) \wedge T_{n}^{K}}^{\prime \prime}-\pi_{\tau \wedge T_{n}^{K}}^{\prime \prime}\right]-\left(e^{r}-1\right)\left[A_{(\tau+\delta) \wedge T_{n}^{K}}^{\prime \prime}-A_{\tau \wedge T_{n}^{K}}^{\prime \prime}\right] \\
& \geq K\left(r \eta-\left(e^{r}-1\right) \delta \mathfrak{b}(n+1)^{2}\right) .
\end{aligned}
$$

Now, with $\delta$ small enough and $r=\log \left(\frac{\eta}{\delta 6(n+1)^{2}}\right)$,

$$
z_{T+\delta}^{\prime \prime}(\nu) \geq \exp \left(K\left[\eta \log \left(\frac{\eta}{\delta \mathfrak{b}(n+1)^{2}}\right)-\eta+\mathfrak{b} \delta(n+1)^{2}\right]\right) .
$$


Hence

$$
\begin{aligned}
& \frac{1}{K} \log \sup _{\tau \leq T} P\left(\pi_{(\tau+\delta) \wedge T_{n}^{K}}^{\prime \prime}-\pi_{\tau \wedge T_{n}^{K}}^{\prime \prime}>K \eta\right) \\
& \quad \leq-\eta \log \left(\frac{\eta}{\delta \mathfrak{b}(n+1)^{2}}\right)-\eta+\mathfrak{b} \delta(n+1)^{2} \rightarrow-\infty, \quad \delta \rightarrow 0 .
\end{aligned}
$$

A.4. C-Local-LDP. Upper bound. Obviously, for $\phi_{0} \neq x_{0}$ or $\psi_{0} \neq y_{0}$ the upper bound is equal to $-\infty$. The proof that for $\phi_{0}=x_{0}$ and $\psi_{0}=y_{0}$ and $(\phi, \psi) \in \mathbb{D}_{[0, T]}^{2} \backslash \mathbb{C}_{[0, T]}^{2}$ the upper bound is equal to $-\infty$ under the $C$-exponential tightness can be found in Theorem 1.3 in Liptser and Pukhalskii (1992).

Thus, the proof is concentrated on the case $(\phi, \psi) \in \mathbb{C}_{[0, T]}^{2}$ with $\phi_{0}=x_{0}$, $\psi_{0}=y_{0}$. Let $Z_{t}^{K}\left(\frac{\lambda}{K}, \frac{\mu}{K}\right)$ be defined in (2.13) with piecewise constant (deterministic) functions $\lambda(t)$ and $\mu(t)$. Since $E Z_{T}^{K}(K \lambda, K \mu) \leq 1$, write

$$
1 \geq E Z_{T}^{K}\left(\frac{\lambda}{K}, \frac{\mu}{K}\right) I\left(\varrho\left(\left(x^{K}, y^{K}\right),(\phi, \psi)\right) \leq \delta\right) .
$$

On the set $\left\{\varrho\left(\left(x^{K}, y^{K}\right),(\phi, \psi)\right) \leq \delta\right\}$, we evaluate $Z_{t}^{K}\left(\frac{\lambda}{K}, \frac{\mu}{K}\right)$ from below. Since $\lambda, \mu$ are piecewise constant functions notations, $\int_{0}^{T} \lambda(t) d \phi_{t} \int_{0}^{T} \mu(t) d \psi_{t}$ will be used for

$$
\sum_{t_{j} \leq T} \lambda\left(t_{j-1}\right)\left[\phi_{t_{j}}-\phi_{t_{j-1}}\right] \quad \text { and } \quad \sum_{t_{i} \leq T} \mu\left(t_{i-1}\right)\left[\psi_{t_{i}}-\psi_{t_{i-1}}\right],
$$

respectively. Obviously, a positive constant $c$ can be chosen such that

$$
\begin{aligned}
\log Z_{T}^{K}\left(\frac{\lambda}{K}, \frac{\mu}{K}\right) \\
\quad \geq-K\left(c \delta+\int_{0}^{T}\left(\lambda(t) d \phi_{t}+\mu(t) d \psi_{t}-G\left(\lambda(t), \mu(t) ; \phi_{t}, \psi_{t}\right) d t\right)\right) .
\end{aligned}
$$

Therefore, this lower bound jointly with (A.11) imply

$$
\begin{aligned}
& \underset{\delta \rightarrow 0}{\lim \sup } \limsup _{K \rightarrow \infty} \frac{1}{K} \log P\left(\varrho\left(\left(x^{K}, y^{K}\right),(\phi, \psi)\right) \leq \delta\right) \\
& \quad \leq-\int_{0}^{T}\left(\lambda(t) d \phi_{t}+\mu(t) d \psi_{t}-G\left(\lambda(t), \mu(t) ; \phi_{t}, \psi_{t}\right) d t\right) .
\end{aligned}
$$

Since the left side of this inequality is independent of $\lambda(t), \mu(t)$ the required upper bound is obtained by minimization of the right side in $\lambda(t), \mu(t)$. As in Liptser and Pukhalskii [(1992), Theorem 6.1], we find that for not absolutely continuous $\phi_{t}$ or $\psi_{t}$ the minimal value of the right-hand side is equal to $-\infty$, while if both $\phi_{t}, \psi_{t}$ are absolutely continuous functions the minimal value coincides with $-J_{T}(\phi, \psi)$. 
A.5. C-Local-LDP. Lower bound. It suffices to verify (A.4) only for $J_{T}(\phi$, $\psi)<\infty$. That supposes the verification of (A.4) for absolutely continuous functions $\left(\phi_{t}, \psi_{t}\right)_{t \leq T}$ from $\mathbb{D}_{[0, T]}^{2,+}$ with $\phi_{0}=x_{0}, \psi_{0}=y_{0}$. Moreover, to satisfy $J_{T}(\phi, \psi)<\infty$ functions $\phi_{t}, \phi_{t}$ have to be absorbed on axis "x $=0$ "; " $\mathrm{y}=0$ " (recall that $x_{t}^{K}, y_{t}^{K}$ are absorbed on these axes). For definiteness we denote the class of the above-mentioned functions $\phi_{t}, \psi_{t}$ by $\mathfrak{A}$.

Set $T^{\phi}=\inf \left\{t>0: \phi_{t}=0\right\}$ and $T^{\psi}=\inf \left\{t>0: \psi_{t}=0\right\}(\inf \{\varnothing\}=\infty)$ and introduce

$$
\begin{aligned}
& J_{T}^{\phi}(\phi, \psi)=\int_{0}^{T^{\phi} \wedge T} \sup _{\lambda}\left(\lambda \dot{\phi}_{t}-G_{\phi}\left(\lambda ; \phi_{t}, \psi_{t}\right) d t\right), \\
& J_{T}^{\psi}(\phi, \psi)=\int_{0}^{T^{\psi} \wedge T} \sup _{\mu}\left(\mu \dot{\psi}_{t}-G_{\psi}\left(\mu ; \phi_{t}, \psi_{t}\right) d t\right) .
\end{aligned}
$$

Then, obviously, we have $J_{T}(\phi, \psi)=J_{T}^{\phi}(\phi, \psi)+J_{T}^{\psi}(\phi, \psi)$. Below we give detailed description of $\lambda^{*}(t)=\operatorname{argmax}_{\lambda}\left(\lambda \dot{\phi}_{t}-G_{\phi}\left(\lambda ; \phi_{t}, \psi_{t}\right)\right)$ and $\mu^{*}(t)=$ $\operatorname{argmax}_{\mu}\left(\mu \dot{\psi}_{t}-G_{\psi}\left(\mu ; \phi_{t}, \psi_{t}\right)\right)$ for $T^{\phi} \wedge T^{\psi}=\infty$,

$$
\begin{aligned}
\lambda^{*}(t) & =\log \left(\frac{\dot{\phi}_{t}}{2 \mathfrak{a} \phi_{t}}+\sqrt{\frac{\dot{\phi}_{t}^{2}}{4\left(\mathfrak{a} \phi_{t}\right)^{2}}+\frac{\mathfrak{b} \psi_{t}}{\mathfrak{a}}}\right), \\
\mu^{*}(t) & =\log \left(\frac{\dot{\psi}_{t}}{2 \mathfrak{c} \phi_{t} \psi_{t}}+\sqrt{\frac{\dot{\psi}_{t}^{2}}{4\left(\mathfrak{c} \phi_{t} \psi_{t}\right)^{2}}+\frac{\mathfrak{D}}{\mathfrak{c} \phi_{t}}}\right) .
\end{aligned}
$$

\section{A.5.1. Main Lemma.}

LeMma 3. Assume

(i) $(\phi, \psi) \in \mathfrak{X}$.

(ii) $T^{\phi} \wedge T^{\psi}=\infty$.

(iii) $\dot{\phi}_{t} \leq$ const., $\dot{\psi}_{t} \leq$ const.

Then there is a positive constant $L(\phi, \psi)$, depending on $L(\phi, \psi)$, such that for $\delta>0$ small enough,

$$
\liminf _{K \rightarrow \infty} \frac{1}{K} \log P\left(\varrho\left(\left(x^{K}, y^{K}\right),(\phi, \psi)\right) \leq \delta\right) \geq-J_{T}(\phi, \psi)-L(\phi, \psi) \delta
$$

Proof. Set $\tau_{\phi}^{K}=\inf \left\{t:\left|x_{t}^{K}-\phi_{t}\right| \geq \delta\right\}$ and $\tau_{\psi}^{K}=\inf \left\{t:\left|y_{t}^{K}-\psi_{t}\right| \geq \delta\right\}$. Since jumps of $x_{t}^{K}, y_{t}^{K}$ are bounded by $\frac{1}{K}$, by the triangular inequality we have $\left|x_{t}^{K}-\phi_{t}\right| \leq\left|x_{t-}^{K}-\phi_{t}\right|+\frac{1}{K}$ and $\left|y_{t}^{K}-\psi_{t}\right| \leq\left|y_{t-}^{K}-\psi_{t}\right|+\frac{1}{K}$. Consequently 
with $K \geq \frac{\delta}{4}$ the inclusion holds:

$$
\begin{aligned}
\left\{\sup _{t \leq T}\left|x_{t}^{K}-\phi_{t}\right|+\sup _{t \leq T}\left|y_{t}^{K}-\psi_{t}\right| \leq \delta\right\} & \\
& \supseteq\left\{\sup _{t \leq T}\left|x_{t-}^{K}-\phi_{t}\right|+\sup _{t \leq T}\left|y_{t-}^{K}-\psi_{t}\right| \leq \frac{\delta}{2}\right\} \\
& =\left\{\sup _{t \leq \tau_{\phi}^{K} \wedge T}\left|x_{t-}^{K}-\phi_{t}\right|+\sup _{t \leq \tau_{\psi}^{K} \wedge T}\left|y_{t-}^{K}-\psi_{t}\right| \leq \frac{\delta}{2}\right\} .
\end{aligned}
$$

Hence, the first lower bound we use is the following:

$$
\begin{aligned}
& P\left(\varrho\left(\left(x^{K}, y^{K}\right),(\phi, \psi)\right) \leq \delta\right) \\
& \quad \geq P\left(\sup _{t \leq \tau_{\phi}^{K} \wedge T}\left|x_{t-}^{K}-\phi_{t}\right|+\sup _{t \leq \tau_{\psi}^{K} \wedge T}\left|y_{t-}^{K}-\psi_{t}\right| \leq \frac{\delta}{2}\right) .
\end{aligned}
$$

For $K$ large enough set

$$
\lambda^{K}(t-)=\log \left(\frac{\dot{\phi}_{t}}{2 \mathfrak{a} x_{t-}^{K}}+\sqrt{\frac{\dot{\phi}_{t}^{2}}{\left(\mathfrak{a} x_{t-}^{K}\right)^{2}}+\frac{\mathfrak{b} x_{t-}^{K} y_{t-}^{K}}{\mathfrak{a} x_{t-}^{K}}}\right), \quad t \leq \tau_{\phi}^{K} \wedge T,
$$

$$
\mu^{K}(t-)=\log \left(\frac{\dot{\psi}_{t}}{2 \mathfrak{c} x_{t-}^{K} y_{t-}^{K}}+\sqrt{\frac{\dot{\psi}_{t}^{2}}{\left(2 \mathfrak{c} x_{t-}^{K} y_{t-}^{K}\right)^{2}} \frac{\grave{D} y_{t-}^{K}}{\mathfrak{c} x_{t-}^{K} y_{t-}^{K}}}\right), \quad t \leq \tau_{\psi}^{K} \wedge T .
$$

$\left[\lambda^{K}(t), \mu^{K}(t)\right.$ are defined similarly with an obvious change]. It is clear that for $K$ large enough $\lambda^{K}(t) ; \mu^{K}(t)$ are bounded and strictly positive.

We define now the positive supermartingale [compare (2.13)]

$$
\begin{aligned}
Z_{t}^{K}\left(\lambda^{K}, \mu^{K}\right)= & \exp \left\{K \int_{0}^{t \wedge \tau_{\phi}^{K} \wedge T}\left[\lambda^{K}(t-) d x_{t}^{K}-G_{\phi}\left(\lambda^{K}(t) ; x_{t}^{K}, y_{t}^{K}\right) d t\right]\right\} \\
& \times \exp \left\{K \int_{0}^{t \wedge \tau_{\psi}^{K} \wedge T}\left[\mu^{K}(t-) d y_{t}^{K}-G_{\psi}\left(\mu^{K}(t) ; x_{t}^{K}, y_{t}^{K}\right) d t\right]\right\} .
\end{aligned}
$$

We show that $\left(Z_{t}^{K}\left(\lambda^{K}, \mu^{K}\right)\right)_{t \leq T}$ is the square integrable martingale with

$$
E Z_{T}^{K}\left(\lambda^{K}, \mu^{K}\right)=1 .
$$

To prove this property we have to check that $E\left(Z_{T}^{K}\left(\lambda^{K}, \mu^{K}\right)\right)^{2}<\infty$. (A.17) implies

$$
\begin{aligned}
& \left(Z_{T}^{K}\left(\lambda^{K}, \mu^{K}\right)\right)^{2}=Z_{T}^{K}\left(2 \lambda^{K}, 2 \mu^{K}\right) \\
& \quad \times \exp \left\{K \int_{0}^{\tau_{\phi}^{K} \wedge T}\left[G_{\phi}\left(2 \lambda^{K}(t) ; x_{t}^{K}, y_{t}^{K}\right)-2 G_{\phi}\left(\lambda^{K}(t) ; x_{t}^{K}, y_{t}^{k}\right)\right] d t\right\} \\
& \quad \times \exp \left\{K \int_{0}^{\tau_{\phi}^{K} \wedge T}\left[G_{\psi}\left(2 \mu^{K}(t) ; x_{t}^{K}, y_{t}^{K}\right)-2 G_{\psi}\left(\mu^{K}(t) ; x_{t}^{K}, y_{t}^{k}\right)\right] d t\right\} .
\end{aligned}
$$


The process $\left(Z_{T}^{K}\left(2 \lambda^{K}, 2 \mu^{K}\right)\right)_{t \leq T}$ is a supermartingale as well; that is

$$
E Z_{T}^{K}\left(2 \lambda^{K}, 2 \mu^{K}\right) \leq 1,
$$

and at the same time under assumptions of the lemma $G_{\phi}\left(2 \lambda^{K}(t) ; x_{t}^{K}, y_{t}^{K}\right)$, $G_{\phi}\left(\lambda^{K}(t) ; x_{t}^{K}, y_{t}^{K}\right)$ and $G_{\psi}\left(2 \mu^{K}(t) ; x_{t}^{K}, y_{t}^{K}\right), G_{\psi}\left(\mu^{K}(t) ; x_{t}^{K}, y_{t}^{K}\right)$ are bounded functions on the time intervals $\left[0, \tau_{\phi}^{K} \wedge T\right]$ and $\left[0, \tau_{\psi}^{K} \wedge T\right]$, respectively. Consequently, $\left(Z_{T}^{K}\left(\lambda^{K}, \mu^{K}\right)\right)^{2}$ has a finite expectation.

Now, $\left(\Omega, \mathscr{F}, \mathbf{F}=\left(\mathscr{F}_{t}\right)_{t \geq 0}, Q^{K}\right)$ with $d Q^{K}=Z_{T}^{K}\left(\lambda^{K}, \mu^{K}\right) d P$ is the stochastic basis. Due to the positiveness of $Z_{T}^{K}\left(\lambda^{K}, \mu^{K}\right)$ not only $Q^{K} \ll P$ but also $P \ll$ $Q^{K}$ with $d P=\left(Z_{T}^{K}\left(\lambda^{K}, \mu^{K}\right)\right)^{-1} d Q^{K}$. We apply this formula for the right side of (A.15). With the notation

$$
\mathfrak{B}_{\delta}^{K}=\left\{\sup _{t \leq \tau_{\phi}^{K} \wedge T}\left|x_{t-}^{K}-\phi_{t}\right|+\sup _{t \leq \tau_{\psi}^{K} \wedge T}\left|y_{t-}^{K}-\psi_{t}\right| \leq \frac{\delta}{2}\right\}
$$

we have

$$
P\left(\mathfrak{B}_{\delta}^{K}\right)=\int_{\mathfrak{B}_{\delta}^{K}}\left(Z_{T}^{K}\left(\lambda^{K}, \mu^{K}\right)\right)^{-1} d Q^{K} .
$$

The random process $Z_{t \wedge T}^{K}\left(\lambda^{K}, \mu^{K}\right)$ is the martingale with respect to $P$ and since $P \sim Q^{K}$ it is a semimartingale with respect to $Q^{K}$ [see, e.g., Liptser and Shiryayev (1989), Chapter 4, Section 5]. For further analysis $Q^{K}$ semimartingale description of $Z_{t \wedge T}^{K}\left(\lambda^{K}, \mu^{K}\right)$ is required. To this end,we use the fact that $P$-semimartingales $x_{t}^{K}, y_{T}^{K}$ are $Q^{K}$ semimartingales as well [see Liptser and Shiryayev (1989), Chapter 4, Section 5] and find their semimartingale decompositions. Let us note that $\pi_{t}^{\prime}, \pi_{t}^{\prime \prime}, \hat{\pi}_{t}^{\prime}, \hat{\pi}_{t}^{\prime \prime}$ are counting processes with respect to both measures $P$ and $Q^{K}$. Recall that $A_{t}^{\prime}, A_{t}^{\prime \prime}, \widehat{A}_{t}^{\prime}, \widehat{A}_{t}^{\prime \prime}$ are their compensators with respect to $P$ and denote by $A_{t}^{\prime Q}, A_{t}^{\prime \prime Q}, \widehat{A}_{t}^{\prime Q}, \widehat{A}_{t}^{\prime \prime Q}$ their compensators with respect to $Q^{K}$. Then the $P$-martingale $m_{t}^{K}=\frac{1}{K}\left(\left[\pi_{t}^{\prime}-A_{t}^{\prime}\right]-\left[\pi_{t}^{\prime \prime}-A_{t}^{\prime \prime}\right]\right)$ obeys the semimartingale decomposition ( $P$-and $Q^{K}$-a.s.)

$$
\begin{aligned}
m_{t}^{K}= & \frac{1}{K}\left(\left[\pi_{t}^{\prime}-A_{t}^{\prime Q}\right]-\left[\pi_{t}^{\prime \prime}-A_{t}^{\prime \prime Q}\right]\right) \\
& -\frac{1}{K}\left(\left[A_{t}^{\prime}-A_{t}^{\prime Q}\right]-\left[A_{t}^{\prime \prime}-A_{t}^{\prime \prime Q}\right]\right) \\
= & m_{t}^{K, Q}-\frac{1}{K}\left(\left[A_{t}^{\prime}-A_{t}^{\prime Q}\right]-\left[A_{t}^{\prime \prime}-A_{t}^{\prime \prime Q}\right]\right)
\end{aligned}
$$

with $Q^{K}$ - square integrable martingales $m_{t}^{K, Q}$ with the predicatable quadratic variation $\left\langle m^{K, Q}\right\rangle_{t}=\frac{1}{K}\left(A_{t}^{\prime Q}+A_{t}^{\prime \prime Q}\right)$. Analogously, the $Q^{K}$-semimartingale decomposition $\hat{m}_{t}^{K}=\hat{m}_{t}^{K, Q}-\frac{1}{K}\left(\left[\widehat{A}_{t}^{\prime}-\widehat{A}_{t}^{\prime Q}\right]-\left[\widehat{A}_{t}^{\prime \prime}-\widehat{A}_{t}^{\prime \prime Q}\right]\right)$ holds with the square integrable martingale $\hat{m}_{t}^{K, Q}$ with $\left\langle\hat{m}^{K, Q}\right\rangle_{t}=\frac{1}{K}\left(\widehat{A}_{t}^{\prime Q}+\widehat{A}_{t}^{\prime \prime Q}\right)$ and $\left\langle m^{K, Q}\right.$, $\left.\hat{m}^{K, Q}\right\rangle_{t} \equiv 0$ (recall that disjointness for jumps of counting processes remains valid with respect to $Q^{K}$ ). 
We give now formulas for $A_{t}^{\prime Q}, A_{t}^{\prime \prime Q}, \widehat{A}_{t}^{\prime Q}, \widehat{A}_{t}^{\prime \prime Q}$. With $\Delta \pi_{t}^{\prime}=\pi_{t}^{\prime}-\pi_{t-1}^{\prime}, \ldots$, $\Delta \hat{\pi}_{t}^{\prime \prime}=\hat{\pi}_{t}^{\prime \prime}-\hat{\pi}_{t}^{\prime \prime}$ we have

$$
\begin{aligned}
Z_{t}^{K}\left(\lambda^{K}, \mu^{K}\right)= & X_{t-}^{K}\left(\lambda^{K}, \mu^{K}\right) \exp \left(\lambda^{K}(t-)\left[\Delta \pi_{t}^{\prime}-\Delta \pi_{T}^{\prime \prime}\right]\right. \\
& \left.+\mu^{K}(t-)\left[\Delta \hat{\pi}_{t}^{\prime}-\Delta \hat{\pi}_{t}^{\prime \prime}\right]\right) .
\end{aligned}
$$

$\left(A_{t}^{\prime Q}\right)$ : It is well known that $A_{t}^{\prime Q}$ is defined by integral equation: for any bounded and predictable function $u(t, \omega)$,

$$
\int_{\Omega} \int_{0}^{T} u(t, \omega) d \pi_{t}^{\prime} d Q^{K}=\int_{\Omega} \int_{0}^{T} u(t, \omega) d A_{t}^{\prime Q} d Q^{K}
$$

Taking into account that $d Q^{K}=Z_{T}^{K}\left(\lambda^{K}, \mu^{K}\right) P$ and $Z_{t \wedge T}^{K}\left(\lambda^{K}, \mu^{K}\right)$ is the martingale with respect to $P$ we get

$$
\begin{aligned}
\int_{\Omega} \int_{0}^{T} u(t, \omega) d \pi_{t}^{\prime \prime} d Q^{K} & =\int_{\Omega} \int_{0}^{T} u(t, \omega) Z_{T}^{K}\left(\lambda^{K}, \mu^{K}\right) d \pi_{t}^{\prime} d P \\
& =\int_{\Omega} \int_{0}^{T} u(t, \omega) Z_{t}^{K}\left(\lambda^{K}, \mu^{K}\right) d \pi_{t}^{\prime} d P .
\end{aligned}
$$

Since jumps of counting processes are disjoint the right side of the above equality is equal to [see (A.20)] $\int_{\Omega} \int_{0}^{T} u(t, \omega) Z_{t-}^{K}\left(\lambda^{K}, \mu^{K}\right) e^{\lambda^{K}(t-) \Delta \pi_{t}^{\prime}} d \pi_{t}^{\prime} d P$ or, what is the same, $\int_{\Omega} \int_{0}^{T} u(t, \omega) Z_{t-}^{K}\left(\lambda^{K}, \mu^{K}\right) e^{\lambda^{K}(t-)} d \pi_{t}^{\prime} d P$. Now, since $Z_{t-}^{K}\left(\lambda^{K}, \mu^{K}\right)$ is the predictable process, the latter integral coincides with

$$
\int_{\Omega} \int_{0}^{T} u(t, \omega) Z_{t-}^{K}\left(\lambda^{K}, \mu^{K}\right) e^{\lambda^{K}(t-)} d A_{t}^{\prime} d P
$$

which, due to the martingale property of $Z_{t}^{K}\left(\lambda^{K}, \mu^{K}\right)$, is equal to

$$
\int_{\Omega} \int_{0}^{T} u(t, \omega) Z_{T}^{K}\left(\lambda^{K}, \mu^{K}\right) e^{\lambda^{K}(t-)} d A_{t}^{\prime} d P:=\int_{\Omega} \int_{0}^{T} u(t, \omega) e^{\lambda^{K}(t-)} d A_{t}^{\prime} d Q^{K} .
$$

Consequently,

$$
d A_{t}^{\prime Q}=e^{\lambda^{K}(t-)} d A_{t}^{\prime}
$$

and $d A_{t}^{\prime \prime Q}=e^{-\lambda^{K}(t-)} d A_{t}^{\prime \prime}, d \widehat{A}_{t}^{\prime Q}=e^{\mu^{K}(t-)} d \widehat{A}_{t}^{\prime}, d \widehat{A}_{t}^{\prime \prime Q}=e^{-\mu^{K}(t-)} d \widehat{A}_{t}^{\prime \prime}$ are obtained in the same way.

Hence

$$
\begin{aligned}
& d m_{t}^{K}=d m_{t}^{K, Q}+\left(\left(e^{\lambda^{K}(t)}-1\right) \mathfrak{a} x_{t}^{K}-\left(e^{-\lambda^{K}(t)}-1\right) \mathfrak{b} x_{t}^{K} y_{t}^{K}\right) d t, \\
& d \hat{m}_{t}^{K}=d \hat{m}_{t}^{K, Q}+\left(\left(e^{\mu^{K}(t)}-1\right) \mathfrak{c} x_{t}^{K} y_{t}^{K}-\left(e^{-\mu^{K}(t)}-1\right) \mathfrak{b} y_{t}^{K}\right) d t
\end{aligned}
$$


and thereby we find the semimartingale decomposition with respect to $Q^{K}$ :

$$
\begin{aligned}
d x_{t}^{K}=\{ & x_{t}^{K}\left(\mathfrak{a}-\mathfrak{b} y_{t}^{K}\right) \\
& \left.+\left(\left(e^{\lambda^{K}(t)}-1\right) \mathfrak{a} x_{t}^{K}-\left(e^{-\lambda^{K}(t)}-1\right) \mathfrak{b} x_{t}^{K} y_{t}^{K}\right)\right\} d t+d m_{t}^{K, Q} \\
= & \frac{\partial}{\partial \lambda} G_{\phi}\left(\lambda^{K}(t) ; x_{t}^{K}, y_{t}^{K}\right) d t+d m_{t}^{K, Q}=\dot{\phi}_{t} d t+d m_{t}^{K, Q} \\
d y_{t}^{K}= & \left\{y_{t}^{K}\left(\mathfrak{c} x_{t}^{K}-\mathfrak{b}\right)\right. \\
& \left.\quad+\left(\left(e^{\mu^{K}(t)}-1\right) \mathfrak{c} x_{t}^{K} y_{t}^{K}-\left(e^{-\mu^{K}(t)}-1\right) \mathfrak{b} y_{t}^{K}\right)\right\} d t+d \hat{m}_{t}^{K, Q}, \\
= & \frac{\partial}{\partial \mu} G_{\psi}\left(\mu^{K}(t) ; x_{t}^{K}, y_{t}^{K}\right) d t+d \hat{m}_{t}^{K, Q}=\dot{\psi}_{t} d t+d \hat{m}_{t}^{K, Q} .
\end{aligned}
$$

The use of this decomposition allows to obtain a description for $\frac{1}{K} \log Z_{T}^{K}\left(\lambda^{K}\right.$, $\mu^{K}$ ) with respect to $Q^{K}$ :

$$
\begin{aligned}
& \frac{1}{K} \log Z_{T}^{K}\left(\lambda^{K}, \mu^{K}\right) \\
& =\int_{0}^{\tau_{\phi}^{K} \wedge T} \lambda^{K}(t-) d m_{t}^{K, Q}+\int_{0}^{\tau_{\phi}^{K} \wedge T}\left(\lambda^{K}(t) \dot{\phi}_{t}-G_{\phi}\left(\lambda^{K}(t) ; x_{t}^{K}, y_{t}^{K}\right)\right) d t \\
& \quad+\int_{0}^{\tau_{\psi}^{K} \wedge T} \mu^{K}(t-) d \hat{m}_{t}^{K, Q}+\int_{0}^{\tau_{\psi}^{K} \wedge T}\left(\mu^{K}(t) \dot{\psi}_{t}-G_{\psi}\left(\mu^{K}(t) ; x_{t}^{K}, y_{t}^{K}\right)\right) d t .
\end{aligned}
$$

Let us estimate above the right side of this equality on the set $\mathfrak{B}_{\delta}^{K}$. There are positive constants $c_{\phi}$ and $c_{\psi}$ so that

$$
\begin{aligned}
& \sup _{t \leq \tau_{\phi}^{K} \wedge T}\left|\lambda^{K}(t)-\lambda^{*}(t)\right|+\sup _{t \leq \tau_{\phi}^{K} \wedge T}\left|G_{\phi}\left(\lambda^{K}(t) ; x_{t}^{K}, y_{t}^{K}\right)-G_{\phi}\left(\lambda^{*}(t) ; \phi_{t}, \psi_{t}\right)\right| \leq c_{\phi} \delta, \\
& \sup _{t \leq \tau_{\psi}^{K} \wedge T}\left|\mu^{K}(t)-\mu^{*}(t)\right|+\sup _{t \leq \tau_{\psi}^{K} \wedge T}\left|G_{\psi}\left(\mu^{K}(t) ; x_{t}^{K}, y_{t}^{K}\right)-G_{\psi}\left(\mu^{*}(t) ; \phi_{t}, \psi_{t}\right)\right| \leq c_{\psi} \delta .
\end{aligned}
$$

Further, since

$$
\begin{aligned}
& \lambda^{*}(t) \dot{\phi}_{t}-G_{\phi}\left(\lambda^{*}(t) ; \phi_{t}, \psi_{t}\right)=\sup _{\lambda}\left(\dot{\phi}_{t}-G_{\phi}\left(\lambda ; \phi_{t}, \psi_{t}\right)\right), \\
& \mu^{*}(t) \dot{\psi}_{t}-G_{\psi}\left(\mu^{*}(t) ; \phi_{t}, \psi_{t}\right)=\sup _{\mu}\left(\dot{\psi}_{t}-G_{\psi}\left(\mu ; \phi_{t}, \psi_{t}\right)\right)
\end{aligned}
$$

and

$$
\begin{aligned}
J_{T}(\phi, \psi)= & \int_{0}^{\tau_{\phi}^{K} \wedge T} \sup _{\lambda}\left(\dot{\phi}_{t}-G_{\phi}\left(\lambda ; \phi_{t}, \psi_{t}\right)\right) d t \\
& +\int_{0}^{\tau_{\psi}^{K} \wedge T} \sup _{\mu}\left(\dot{\psi}_{t}-G_{\psi}\left(\mu ; \phi_{t}, \psi_{t}\right)\right) d t
\end{aligned}
$$

we find

$$
\begin{aligned}
\frac{1}{K} \log Z_{T}^{K}\left(\lambda^{K}, \mu^{K}\right) \leq & \left|\int_{0}^{\tau_{\phi}^{K} \wedge T} \lambda^{K}(t-) d m_{t}^{K, Q}\right|+\left|\int_{0}^{\tau_{\psi}^{K} \wedge T} \mu^{K}(t-) d \hat{m}_{t}^{K, Q}\right| \\
& +\left(c_{\phi}+c_{\psi}\right) T \delta+J_{T}(\phi, \psi) .
\end{aligned}
$$


This upper bound implies the lower one,

$$
\begin{aligned}
Z_{T}^{K}\left(\lambda^{K}, \mu^{K}\right) \geq & \exp \left(-K\left(J_{T}(\phi, \psi)+\left(c_{\phi}+c_{\psi}\right) T \delta\right)\right) \\
& \times \exp \left(-K\left|\int_{0}^{\tau_{\phi}^{K} \wedge T} \lambda^{K}(t-) d m_{t}^{K, Q}\right|-K\left|\int_{0}^{\tau_{\psi}^{K} \wedge T} \mu^{K}(t-) d \hat{m}_{t}^{K, Q}\right|\right),
\end{aligned}
$$

which, being applied in (A.18), gives

$$
\begin{aligned}
& P\left(\mathfrak{B}_{\delta}^{K}\right) \geq \exp \left(-K\left(J(\phi, \psi)+\left(c_{\phi}+c_{\psi}\right) T \delta\right)\right) \\
& \quad \times \int_{\mathfrak{B}_{\delta}^{K}} \exp \left(-K\left|\int_{0}^{\tau_{\phi}^{K}} \lambda^{K}(t-) d m_{t}^{K, Q}\right|\right. \\
&\left.\quad-K\left|\int_{0}^{\tau_{\psi}^{K} \wedge T} \mu^{K}(t-) \hat{m}_{t}^{K, Q}\right|\right) d Q^{K} .
\end{aligned}
$$

(A.22) can be sharpened as

$$
P\left(\mathfrak{B}_{\delta}^{K}\right) \geq e^{-K\left(J_{T}(\phi, \psi)+\left(c_{\phi}+c_{\psi}\right) T \delta+2 \varepsilon\right)} Q^{K}\left(\mathfrak{M}_{\varepsilon}^{K} \cap \mathfrak{N}_{\varepsilon}^{K} \cap \mathfrak{B}_{\delta}^{K}\right),
$$

where

$\mathfrak{M}_{\varepsilon}^{K}=\left\{\left|\int_{0}^{\tau_{\phi}^{K} \wedge T} \lambda^{K}(t-) d m_{t}^{K, Q}\right|<\varepsilon\right\} \quad$ and $\quad \mathfrak{N}_{\varepsilon}^{K}=\left\{\left|\int_{0}^{\tau_{\psi}^{K} \wedge T} \mu^{K}(t-) d \hat{m}_{t}^{K, Q}\right|<\varepsilon\right\}$.

Hence

$$
\begin{aligned}
\liminf _{K \rightarrow \infty} \frac{1}{K} \log P\left(\mathfrak{B}_{\delta}^{K}\right) \geq & -J_{T}\left(\phi, \psi-\left(c_{\phi}+c_{\psi}\right) T \delta-2 \varepsilon\right) \\
& +\liminf _{K \rightarrow \infty} \frac{1}{K} \log Q^{K}\left(\mathfrak{M}_{\varepsilon}^{K} \cap \mathfrak{N}_{\varepsilon}^{K} \cap \mathfrak{B}_{\delta}^{K}\right) .
\end{aligned}
$$

The latter inequality jointly with (A.15) imply the statement of the lemma provided that for fixed $\varepsilon$ and $\delta$ it holds $\liminf \operatorname{in}_{K \rightarrow \infty} Q^{K}\left(\mathfrak{M}_{\varepsilon}^{K} \cap \mathfrak{N}_{\varepsilon}^{K} \cap \mathfrak{B}_{\delta}^{K}\right)=1$. We derive that property from

$$
\text { (A.23) } \lim _{K \rightarrow \infty} Q^{K}\left(\Omega \backslash \mathfrak{M}_{\varepsilon}^{K}\right)=0, \quad \lim _{K \rightarrow \infty} Q^{K}\left(\Omega \backslash \mathfrak{M}_{\varepsilon}^{K}\right)=0, \quad \lim _{K \rightarrow \infty} Q^{K}\left(\Omega \backslash \mathfrak{B}_{\delta}^{K}\right)=0 .
$$

The first part of (A.23) follows from the Chebyshev inequality ( $E^{K}$ is the expectation with respect to $Q^{K}$ ),

$$
\begin{aligned}
Q^{K}\left(\Omega \backslash \mathfrak{M}_{\varepsilon}^{K}\right) & =Q^{K}\left(\left|\int_{0}^{\tau_{\phi}^{K} \wedge T} \lambda^{K}(t-) d m_{t}^{K, Q}\right| \geq \varepsilon\right) \\
& \leq \frac{1}{\varepsilon^{2}} E^{K} \int_{0}^{\tau_{\phi}^{K} \wedge T}\left(\lambda^{K}(t)\right)^{2} d\left\langle m^{K, Q}\right\rangle_{t} \\
& \leq \frac{1}{K^{2} \varepsilon^{2}} E^{K} \int_{0}^{\tau_{\phi}^{K} \wedge T}\left(\lambda^{K}(t)\right)^{2}\left(e^{\lambda^{K}(t)} d A_{t}^{\prime}+e^{-\lambda^{K}(t)} d A_{t}^{\prime \prime}\right) \\
& \leq \frac{\text { const. }}{K \varepsilon^{2}} \rightarrow 0, \quad K \rightarrow \infty .
\end{aligned}
$$


The validity of the second part of (A.23) is verified similarly. The third part in (A.23) is verified similarly to the first and second ones since by virtue of (A.21),

$$
\Omega \backslash \mathfrak{B}_{\delta}^{K}=\left\{\sup _{t \leq \tau_{\phi}^{K} \wedge T}\left|m_{t}^{K, Q}\right|+\sup _{t \leq \tau_{\psi}^{K} \wedge T}\left|\hat{m}_{t}^{K, Q}\right| \geq \frac{\delta}{2}\right\} .
$$

\section{A.5.2. The lower bound under $T^{\phi} \wedge T^{\psi}=\infty$.}

LemMa 4. Assume

(i) $(\phi, \psi) \in \mathfrak{A}$.

(ii) $T^{\phi} \wedge T^{\psi}=\infty$.

Then

$$
\liminf _{\delta \rightarrow 0} \liminf _{K \rightarrow \infty} \frac{1}{K} \log P\left(\varrho\left(\left(x^{K}, y^{K}\right),(\phi, \psi)\right) \leq \delta\right) \geq-J_{T}(\phi, \psi) .
$$

Proof. Set $\phi_{t}^{n}=x_{0}+\int_{0}^{r} \dot{\phi}_{s} I\left(\phi_{s} \leq n\right) d s$. Since $\int_{0}^{T}\left|\dot{\phi}_{t}\right| d t<\infty$, it holds

$$
\limsup _{n} \sup _{t \leq T}\left|\phi_{t}-\phi_{t}^{n}\right| \leq \int_{0}^{T} I\left(\dot{\phi}_{t}>n\right)\left|\dot{\phi}_{t}\right| d t=0 .
$$

Similarly, for $\psi_{t}^{n}=y_{0}+\int_{0}^{r} \dot{\psi}_{s} I\left(\psi_{s} \leq n\right) d s$ we have $\lim _{n} \sup _{t \leq T}\left|\psi_{t}-\psi_{t}^{n}\right|=0$. Let us choose $n_{0}$ so that for $n \geq n_{0}$ it holds $\sup _{t \leq T}\left|\phi_{t}-\phi_{t}^{n}\right|+\sup _{t \leq T}\left|\psi_{t}-\psi_{t}^{n}\right| \leq \frac{\delta}{2}$. Since by the triangular inequality,

$$
\sup _{t \leq T}\left|x_{t}^{K}-\phi_{t}\right|+\sup _{t \leq T}\left|y_{t}^{K}-\psi_{t}\right|+\sup _{t \leq T}\left|x_{t}^{K}-\phi_{t}^{n}\right|+\sup _{t \leq T}\left|y_{t}^{K}-\psi_{t}^{n}\right|+\frac{\delta}{2},
$$

for any $n \geq n_{0}$ we get

$$
P\left(\varrho\left(\left(x^{K}, y^{K}\right),(\phi, \psi)\right) \leq \delta\right) \geq P\left(\varrho\left(\left(x^{K}, y^{K}\right),\left(\phi^{n}, \psi^{n}\right)\right) \leq \frac{\delta}{2}\right) .
$$

Therefore, by Lemma 3,

$$
\liminf _{K \rightarrow \infty} \frac{1}{K} \log P\left(\varrho\left(\left(x^{K}, y^{K}\right),\left(\phi^{n}, \psi^{n}\right)\right) \leq \frac{\delta}{2}\right) \geq-J_{T}\left(\phi^{n}, \psi^{n}\right)-L_{\phi^{n}, \psi^{n}} \delta .
$$

Since $\inf _{t \leq T} \phi_{t}>0$ and $\inf _{t \leq T} \psi_{t}>0$ and $\phi_{t}, \psi_{t}$ and approximated by $\phi_{t}^{n}, \psi_{t}^{n}$ uniformly in $t \leq T$, for $n$ large enough a majorant $L_{\phi, \psi}$ for $L_{\phi^{n}, \psi^{n}}$ can be chosen, so that

$$
\liminf _{K \rightarrow \infty} \frac{1}{K} \log P\left(\varrho\left(\left(x^{K}, y^{K}\right),(\phi, \psi)\right) \leq \frac{\delta}{2}\right) \geq-J_{T}\left(\phi^{n}, \psi^{n}\right)-L_{\phi, \psi} \delta .
$$

We show that

$$
\limsup _{n} J_{T}\left(\phi^{n}, \psi^{n}\right) \leq J_{T}(\phi, \psi)
$$


Since for $n$ large enough $\inf _{t \leq T} \phi_{t}^{n}>0, \inf _{t \leq T} \psi_{t}^{n}>0$, introduce

$$
\begin{aligned}
\lambda_{n}^{*}(t) & =\log \left(\frac{\dot{\phi}_{t}^{n}}{2 \mathfrak{a} \phi_{t}^{n}}+\sqrt{\left.\frac{\left(\dot{\phi}^{n}\right)_{t}^{2}}{4\left(\mathfrak{a} \phi_{t}^{n}\right)^{2}}+\frac{\mathfrak{b} \psi_{t}^{n}}{\mathfrak{a}}\right)},\right. \\
\mu^{*}(t) & =\log \left(\frac{\dot{\psi}_{t}^{n}}{2 \mathfrak{c} \phi_{t}^{n} \psi_{t}^{n}}+\sqrt{\frac{\left(\dot{\psi}^{n}\right)_{t}^{2}}{4\left(\mathfrak{c} \phi_{t}^{n} \psi_{t}^{n}\right)^{2}}+\frac{\mathfrak{D}}{\mathfrak{c} \phi_{t}^{n}}}\right)
\end{aligned}
$$

and note that

$$
\begin{aligned}
J_{T}\left(\phi^{n}, \psi^{n}\right)= & \int_{0}^{T} I\left(\dot{\phi}_{t} \leq n\right)\left(\lambda_{n}^{*}(t) \dot{\phi}_{t}-G_{\phi}\left(\lambda_{n}^{*}(t) ; \phi_{t}^{n}, \psi_{t}^{n}\right)\right) d t \\
& +\int_{0}^{T} I\left(\dot{\phi}_{t}>n\right)\left(-G_{\phi}\left(\lambda_{n}^{*}(t) ; \phi_{t}^{n}, \psi_{t}^{n}\right)\right) d t \\
& +\int_{0}^{T} I\left(\dot{\psi}_{t} \leq n\right)\left(\mu_{n}^{*}(t) \dot{\psi}_{t}-G_{\psi}\left(\mu_{n}^{*}(t) ; \phi_{t}^{n}, \psi_{t}^{n}\right)\right) d t \\
& +\int_{0}^{T} I\left(\dot{\psi}_{t}>n\right)\left(-G_{\psi}\left(\mu_{n}^{*}(t) ; \phi^{n}, \psi^{n}\right)\right) d t \\
\leq & J_{T}(\phi, \psi)+\int_{0}^{T}\left|G_{\phi}\left(\lambda^{*}(t) ; \phi_{t}, \psi_{t}\right)-G_{\phi}\left(\lambda_{n}^{*}(t) ; \phi_{t}^{n}, \psi_{t}^{n}\right)\right| d t \\
& +\int_{0}^{T}\left|G_{\psi}\left(\mu^{* \mu}(t) ; \phi_{t}, \psi_{t}\right)-G_{\psi}\left(\lambda_{n}^{*}(t) ; \phi_{t}^{n}, \psi_{t}^{n}\right)\right| d t
\end{aligned}
$$

The second and third terms in the right side of the above inequality converge to zero, as $n \rightarrow \infty$, by virtue of the uniform (in $t \leq T$ ) convergence $\left(\phi^{n}, \psi^{n}\right) \rightarrow$ $(\phi, \psi)$ and $\int_{0}^{T}\left|\dot{\phi}_{t}^{n} \lambda_{n}^{*}(t)-\dot{\phi}_{t} \lambda^{*}(t)\right| d t \rightarrow 0$ and $\int_{0}^{\bar{T}}\left|\dot{\psi}_{t}^{n} \mu_{n}^{*}(t)-\dot{\psi}_{t} \mu^{*}(t)\right| d t \rightarrow 0$; that is, (A.24) holds.

Thus

(A.26) $\liminf _{K \rightarrow \infty} \frac{1}{K} \log P\left(\varrho\left(\left(x^{K}, y^{K}\right),(\phi, \psi)\right) \leq \frac{\delta}{2}\right) \geq-J_{T}(\phi, \psi)-L_{\phi, \psi} \delta$

and the statement of the lemma holds.

A.5.3. The lower bound under $T^{\phi} \wedge T^{\psi}<\infty$. For $(\phi, \psi) \in \mathfrak{A}$ and $h$ small enough we have

$$
\begin{aligned}
& \liminf _{\delta \rightarrow 0} \liminf _{K \rightarrow \infty} \frac{1}{K} \log P\left(\varrho\left(\left(x^{K}, y^{K}\right),(\phi, \psi)\right) \leq \delta\right) \\
& \quad=\liminf _{\delta \rightarrow 0} \liminf _{K \rightarrow \infty} P\left(\sup _{t \leq T^{\phi} \wedge T}\left|x_{t}^{K}-\phi_{t}\right|+\sup _{t \leq T^{\psi} \wedge T}\left|y_{t}^{K}-\psi_{t}\right| \leq \delta\right) \\
& \quad \geq \liminf _{\delta \rightarrow 0} \liminf _{K \rightarrow \infty} P\left(\sup _{t \leq\left(T^{\phi}-h\right) \wedge T}\left|x_{t}^{K}-\phi_{t}\right|+\sup _{t \leq\left(T^{\psi}-h\right) \wedge T}\left|y_{t}^{K}-\psi_{t}\right| \leq \delta\right) .
\end{aligned}
$$


On the other side, for $\phi_{t}^{h} \equiv \phi_{t}, t \leq T^{\phi}-h, \psi_{t}^{h} \equiv \psi_{t}, t \leq T^{\psi}-h$, and for $t>T^{\phi}-h, t>T^{\psi}-h$,

$$
\dot{\phi}_{t}^{h}=\phi_{t}^{h}\left(\mathfrak{a}-\mathfrak{b} \psi_{t}^{h}\right), \quad \dot{\psi}_{t}^{h}=\psi_{t}^{h}\left(\mathfrak{c} \phi_{t}^{h}-\mathfrak{d}\right),
$$

respectively, we have

$$
\begin{aligned}
& \liminf _{\delta \rightarrow 0} \liminf _{K \rightarrow \infty} P\left(\sup _{t \leq\left(T^{\phi}-h\right) \wedge T}\left|x_{t}^{K}-\phi_{t}\right|+\sup _{t \leq\left(T^{\psi}-h\right) \wedge T}\left|y_{t}^{K}-\psi_{t}\right| \leq \delta\right) \\
& \quad=\liminf _{\delta \rightarrow 0} \liminf _{K \rightarrow \infty} P\left(\varrho\left(\left(x^{K}, y^{K}\right),\left(\phi^{h}, \psi^{h}\right)\right) \leq \delta\right) \geq-J_{T}\left(\phi^{h}, \psi^{h}\right),
\end{aligned}
$$

where the latter inequality follows from Lemma 4.

Finally,

$$
\begin{aligned}
J_{Y}\left(\phi^{h}, \psi^{h}\right)= & \int_{0}^{\left(T^{\phi}-h\right) \wedge T} \sup _{\lambda}\left(\lambda \dot{\phi}_{t}-G_{\phi}\left(\lambda ; \phi_{t}, \psi_{t}\right)\right) d t \\
& +\int_{0}^{\left(T^{\psi}-h\right) \wedge T} \sup _{\mu}\left(\mu \dot{\psi}_{t}-G_{\psi}\left(\mu ; \phi_{t}, \psi_{t}\right)\right) d t \\
\leq & \int_{0}^{T^{\phi} \wedge T} \sup _{\lambda}\left(\lambda \dot{\phi}_{t}-G_{\phi}\left(\lambda ; \phi_{t}, \psi_{t}\right)\right) d t \\
& +\int_{0}^{T^{\psi} \wedge T} \sup _{\mu}\left(\mu \dot{\psi}_{t}-G_{\psi}\left(\mu ; \phi_{t}, \psi_{t}\right)\right) d t \\
= & J_{T}(\phi, \psi) .
\end{aligned}
$$

Now combining the obtained results above we arrive at the required lower bound,

$$
\liminf _{\delta \rightarrow 0} \liminf _{K \rightarrow \infty} \frac{1}{K} \log P\left(\varrho\left(\left(x^{K}, y^{K}\right),(\phi, \psi)\right) \leq \delta\right) \geq-J_{T}(\phi, \psi) .
$$

\section{REFERENCES}

Dembo, A. and Zeitouni, O. (1993). Large Deviations Techniques and Applications. Jones and Bartlet, Boston.

Dupuis, P. and ElLIS, R. (1997). A Weak Convergence Approach to the Theory of Large Deviations. Wiley, New York.

FREIDLIN, M. (1998). Random and deterministic perturbation of nonlinear oscillators. Ducumenta Mathematica (Extra Vol.) III 223-235.

Freidlin, M. and Weber, M. (1998). Random perturbation of nonlinear oscillators. Ann. Probab. 26 925-967.

Freidlin, M. I. and Wentzell, A. D. (1984). Random Perturbations of Dynamical Systems. Springer, New York.

Goel, N. S., Maitra, S. C. and Montroll, E. W. (1971). Nonlinear Models for Interacting Populations. Academic Press, New York.

HiтchCOCK, S. E. (1986). Extinction probabilities in predator-prey models. J. Appl. Probab. 23 $1-13$.

KESTEN H. and OGURA Y. (1981). Recurrence properties of Lotka-Volterra models with random fluctuations. J. Math. Soc. Japan 32 335-366. 
KlebaneR, F. C. (1998). Introduction to Stochastic Calculus with Applications. Imperial College Press, London.

KurTZ, T. G. (1981). Approximation of Population Processes. SIAM, Philadelphia.

LiPtSer, R. and ShiryaYev, A. (1978). Statistics of Random Processes 2. Springer, New York.

LIPTSER, R. S. and ShIRYAYEV, A. N. (1989). Theory of Martingales. Kluwer, Dordrecht.

LIPTSER, R. S. and PUKHALSKII, A. A. (1992). Limited theorems on large deviations for semimartingales. Stochastics Stochastics Rep. 38 201-249.

LoTKA, A. J. (1925). Elements of physical Biology. Williams and Wilkins, Baltimore.

May, R. M. (1976). Theoretical Ecology, Principles and Applications. Oxford Univ. Press.

PUKHALSKII, A. A. (1991). On functional principle of large deviations. In New Trends in Probability and Statistics (V. Sazonov and Shervashidze eds.) 198-218. VSP/Mokslas, Vilnius, Lithuania.

PUKHALSKII, A. A. (1999). Large deviations of semimartingales: a maxingale problem approach. II. Uniqueness for the maxingale problem. Applications. Stochastics Stochastic Rep. $\mathbf{6 8}$ 65-143.

Roozen, H. (1989). An asymptotic solution to a two-dimensional exit problem arising in population dynamics. SIAM J. Appl. Math. 49 1793-1810.

SMIth, X. and MEAD, X. (1979). On predicting extinction in simple population models II. Numerical approximations. J. Theoretical Biol. 82 525-535.

Turelli, M. (1977). Random environments and stochastic calculus. Theoret. Population Biol. 12 $140-178$.

VOLTERRA, V. (1926). Variazioni e fluttuazioni del numero d'individui in specie d' animali conviventi. Mem. Acad. Lincei 2 31-113.

WATson, R. (1987). Some thoughts on models for interacting populations. Report 2, Dept. Statistical Research Univ. Melbourne.

Wentzell, A. D. (1989). Limit Theorems of Large Deviation for markov Random Processes. Reidel, Dordrecht.

DEPARTMENT OF MATHEMATICS

AND STATISTICS

UNIVERSITY OF MELBOURNE

PARKVILLE, VICTORIA 3052

AUSTRALIA

E-MAIL: fima@ms.unimelb.edu.au
DEPARTMENT OF ElECTRICAL ENGINEERING SYSTEMS

Tel Aviv UnIVERISTY 69978 Tel AvIV

ISRAEL

E-MAIL: liptser@eng.tau.ac.il 